\title{
Distinct antagonist-bound inactive states underlie the divergence in the structures of the dopamine D2 and D3 receptors
}

\author{
J. Robert Lane ${ }^{1,2^{\star}}$, Ara M. Abramyan³, Ravi Kumar Verma ${ }^{3}$, Herman D. Lim4,

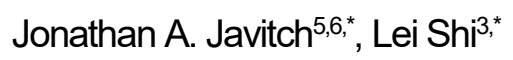

${ }^{1}$ Division of Pharmacology, Physiology and Neuroscience, School of Life Sciences, Queen's Medical Centre, University of Nottingham, Nottingham NG7, 2UH, U.K.

${ }^{2}$ Centre of Membrane Protein and Receptors, Universities of Birmingham and Nottingham, United Kingdom.

${ }^{3}$ Computational Chemistry and Molecular Biophysics Unit, National Institute on Drug Abuse - Intramural Research Program, National Institutes of Health, Baltimore, Maryland, United States

${ }^{4}$ Drug Discovery Biology, Department of Pharmacology and Medicinal Chemistry, Monash Institute of Pharmaceutical Sciences, Monash University (Parkville campus), Parkville, Victoria, Australia

${ }^{5}$ Departments of Psychiatry and Pharmacology, College of Physicians and Surgeons, Columbia University, New York, New York, United States

${ }^{6}$ Division of Molecular Therapeutics, New York State Psychiatric Institute, New York, New York, United States

${ }^{*}$ Corresponding authors:

Email: lei.shi2@nih.gov (LS), Rob.Lane@nottingham.ac.uk (JRL), jaj2@cumc.columbia.edu (JAJ) 


\begin{abstract}
Understanding how crystal structures reflect the range of possible $\mathrm{G}$ protein-coupled receptor (GPCR) states is critical for rational drug discovery (RDD). Combining computational simulations with mutagenesis and binding studies, we find that the structure of the dopamine D2 receptor $\left(D_{2} R\right) /$ risperidone complex captures an inactive receptor conformation that accommodates some but not all antagonist scaffolds. Indeed, we find that eticlopride binds $D_{2} R$ in a configuration very similar to that seen in the $D_{3} R$ structure, in a pose that is incompatible with the $D_{2} R /$ risperidone structure. Moreover, our simulations reveal that extracellular loops 1 and 2 (EL1 and EL2) are highly dynamic, with spontaneous transitions of EL2 from the helical conformation in the $D_{2} R /$ risperidone structure to an extended conformation similar to that in the $D_{3} R /$ eticlopride structure. Our results highlight previously unappreciated conformational diversity and dynamics in the inactive state of a GPCR with potential functional implications. These findings are also of paramount importance for RDD as limiting a virtual screen to one state will miss relevant ligands.
\end{abstract}




\section{INTRODUCTION}

G protein-coupled receptors (GPCRs) are important therapeutic targets for numerous human diseases. Our understanding of GPCR functional mechanisms has evolved from a simple demarcation of single active and inactive states to the appreciation and detection of multiple active states responsible for partial or biased agonism ${ }^{1-3}$. High-resolution crystal structures of these proteins are vital for structure-based (rational) drug discovery (RDD) efforts designed to tailor selectivity and efficacy ${ }^{4,5}$. Indeed, a current focus of the field is on developing functionally biased ligands $^{6-8}$ that couple preferentially to a particular effector pathway. Less attention has been dedicated to the possibility that there may be multiple inactive states, and that different antagonist scaffolds might lead to different receptor conformations. Such a possibility could have major impact on RDD for antagonists, since a crystal structure of a receptor with a particular ligand bound might represent an invalid docking target for an antagonist that binds in a different pose to a different inactive conformation. Although substantial differences in antagonist binding mode and position of the binding pockets have been revealed between different aminergic receptors, no differences in conformation has been detected for the inactive state of any individual aminergic receptor ${ }^{5}$. In particular, although a number of antagonists from different scaffolds have been cocrystallized with $\beta_{2}$ adrenergic receptor, the conformational differences among these crystal structures are minimal ${ }^{5}$.

Curiously, the structures of the highly homologous dopamine D2 and D3 receptors $\left(D_{2} R\right.$ and $D_{3} R$ ) in the inactive states revealed quite substantial differences on the extracellular side of the transmembrane domain when bound with antagonists from different scaffolds ${ }^{9,10}$. Specifically, the $D_{3} R$ structure is in complex with eticlopride, a substituted benzamide (PDB: $\left.3 P B L\right)^{9}$, while the $D_{2} R$ structure is bound with risperidone, a benzisoxazole derivative (PDB: 6CM4) ${ }^{10}$. The binding poses of the two ligands differ substantially. The risperidone is oriented relatively perpendicular to the membrane plane with its benzisoxazole ring penetrating into a hydrophobic pocket beneath the orthosteric binding site (OBS) of $\mathrm{D}_{2} \mathrm{R}$; in contrast, eticlopride is oriented relatively parallel to the 
membrane plane contacting the extracellular portion of TM5 in $D_{3} R$ that risperidone does not touch in $\mathrm{D}_{2} \mathrm{R}^{10,11}$. Nemonapride, another substituted benzamide, binds in the OBS of the slightly divergent $D_{4} R$ (PDB: 5 WIV) ${ }^{12}$ in a manner very similar to that of eticlopride in the $D_{3} R^{11}$. The benzisoxazole moiety of risperidone is enclosed by 8 residues in $D_{2} R$ that are identical among all $D_{2}$-like receptors (i.e., $D_{2} R, D_{3} R$, and $D_{4} R$ ): Cys $118^{3.36}$ (superscripts denote Ballesteros-Weinstein numbering ${ }^{13}$ ), Thr119 ${ }^{3.37}$, Ile122 ${ }^{3.40}$, Ser197 ${ }^{5.46}$, Phe198 ${ }^{5.47}$, Phe382 $2^{6.44}$, Trp386 ${ }^{6.48}$, and Phe390 6.52. Notably, three of these residues on the intracellular side of the OBS, Ile122 ${ }^{3.40}$, Phe198 ${ }^{5.47}$, Phe $382^{6.44}$, accommodate the F-substitution at the tip of the benzisoxazole ring in a small cavity (termed herein as the $I \mathrm{e}^{3.40}$ sub-pocket) (Fig. 1a). Interestingly, this $I \mathrm{l}^{3.40}$ sub-pocket is collapsed in both the $D_{3} R$ and $D_{4} R$ structures ${ }^{11}$ (Fig. 1b,c). We noted that this collapse is associated with rotation of the sidechain of Cys $^{3.36}$ : In the $D_{2} R /$ risperidone structure, the sidechain of Cys ${ }^{3.36}$ faces the OBS, whereas it rotates downwards to partially fill the $l l e^{3.40}$ sub-pocket in the $D_{3}$ R/eticlopride and $\mathrm{D}_{4} \mathrm{R} /$ nemonapride structures.

Importantly, the co-crystalized ligands (risperidone, eticlopride, and nemonapride) display little subtype selectivity across $D_{2}$-like receptors ${ }^{9,12,14,15}$ (also see PDSP database ${ }^{16}$ ). Given the high homology among $D_{2}$-like receptors, especially between $D_{2} R$ and $D_{3} R$ in and near the OBS, we hypothesized that the drastic conformational differences between the structures of these receptors in the inactive state are mostly due to the different binding poses of antagonists of different scaffolds and not to inherent differences between the two receptors. To test this hypothesis, we carried out extensive molecular dynamics (MD) simulations of $D_{2} R$ in complex with non-selective antagonists of different scaffolds to characterize the plasticity of the OBS and the extracellular loop dynamics in the inactive conformational state. 


\section{RESULTS}

In the $D_{2} R$ structure, Ile1223.40, Leu3756.37, and Leu3796.41 were mutated to Ala to thermostabilize the receptor for crystallography. We reverted these thermostabilizing mutations back to their WT residues and established WT $D_{2} R$ models in complex with selected ligands (see Methods, Supplementary Fig. 1 and Supplementary Table 1).

\section{Spiperone but not eticlopride extends into the $11 e^{3.40}$ sub-pocket}

In our MD simulations of the WT $\mathrm{D}_{2} \mathrm{R} /$ risperidone complex, we observed that risperidone stably maintains the binding pose captured in the crystal structure, even without the thermostabilizing mutations (Fig. $1 \mathrm{~d}$ ). Thus, the $1122^{3.40} \mathrm{~A}$ mutation has minimal impact on the binding pose of risperidone. Interestingly in the simulations of the $D_{2} R$ model in complex with spiperone, a butyrophenone derivative, the F-substitution on the butyrophenone ring similarly occupies the $1 \mathrm{e}^{3.40}$ sub-pocket as risperidone (Fig. 1e). Note that the F-substitutions in risperidone and spiperone are located at similar distances to the protonated $\mathrm{N}$ atoms that interact with $\mathrm{Asp}^{3.32}$ (measured by the number of carbon atoms between them, Supplementary Fig. 1) and these two ligands appear to be optimized to occupy the $1 e^{3.40}$ sub-pocket.

In contrast, in our simulations of the $\mathrm{D}_{2} \mathrm{R} /$ eticlopride complex, the eticlopride pose revealed in the $D_{3} R$ structure (PDB: $3 P B L$ ) is stable throughout the simulations and does not protrude into the $1 \mathrm{e}^{3.40}$ sub-pocket (Fig. 1f). Consistent with the difference in the crystal structures noted above (Fig. 1a,b), when risperidone and spiperone occupy the $11 \mathrm{e}^{3.40}$ sub-pocket, the sidechain of Cys $118^{3.36}$ rotates away with its $X^{1}$ rotamer in gauche-, while in the presence of the bound eticlopride, this rotamer is in trans (Supplementary Fig. 2). 


\section{Mutagenesis of $1 /{ }^{122} 2^{3.40}$ and the sensitivity to $\mathrm{Na}^{+}$}

To validate these computational findings, we mutated Ile $122^{3.40}$ of WT $D_{2} R$ to both Trp and Ala and characterized how these mutations would affect the binding affinities for spiperone, risperidone, and eticlopride (Table 1). We hypothesized that the bulkier sidechain of Trp at position 3.40 would hamper the binding of spiperone and risperidone since they occupy the lle 3.40 sub-pocket but have no effect on eticlopride binding, while the smaller Ala should not affect the binding of spiperone or risperidone. Consistent with this hypothesis, compared to WT, the I122W mutation significantly decreased the binding affinities of risperidone and spiperone but had no effect on that of eticlopride. In contrast, the I122A mutation did not affect the affinities of spiperone or risperidone but caused a 3-fold increase in the affinity of eticlopride. This is consistent with our simulation results that show the $1122^{3.40} \mathrm{~A}$ mutation has minimal impact on risperidone binding. Both Ile $122^{3.40}$ and Phe $382^{6.44}$ of the Ile122 $2^{3.40}$ sub-pocket are part of the conserved Pro ${ }^{5.50}-11 e^{3.40}$ Phe ${ }^{6.44}$ motif that undergoes rearrangement upon receptor activation ${ }^{17}$, and we have found that the I122A mutation renders the receptor non-functional ${ }^{10,18}$. Thus, the I122A mutation may promote an inactive conformation of $D_{2} R$ that favors eticlopride binding but has no effect on the binding of risperidone or spiperone. This is consistent with our proposal that different antagonist scaffolds may favor distinct inactive conformations of $D_{2} R$.

We have previously shown that the binding of structurally distinct antagonists to the OBS is differentially modulated by the $\mathrm{Na}^{+}$bound in a conserved allosteric binding pocket coordinated by $\mathrm{Asp}^{2.50}$ and $\mathrm{Ser}^{3.39}$. While spiperone binding was insensitive to the presence of $\mathrm{Na}^{+}$, the affinities of eticlopride and sulpiride were increased and the affinity of zotepine was decreased ${ }^{19}$. In our current simulations, the occupancy of the $1 \mathrm{e}^{3.40}$ sub-pocket by both spiperone and risperidone was unaffected by the presence or absence of bound $\mathrm{Na}^{+}$(Supplementary Fig. 2). Note that Ser ${ }^{3.39}$ and $I l \mathrm{e}^{3.40}$ are adjacent to each other, and we hypothesized that the occupation of the $I \mathrm{e}^{3.40}$ subpocket by spiperone or risperidone may confer a lack of $\mathrm{Na}^{+}$sensitivity to the binding of these inverse agonists. To further test this hypothesis and to understand how $\mathrm{Na}^{+}$influences risperidone 
binding, we performed binding experiments in the absence or presence of $\mathrm{Na}^{+}$and found its affinity to be unaffected (Table 1).

Interestingly in our simulations, while the eticlopride pose is highly stable in the presence of bound $\mathrm{Na}^{+}$, eticlopride oscillated between subtly different poses in the absence of $\mathrm{Na}^{+}$. These oscillations are associated with the sidechain of Cys ${ }^{3.36}$ swinging back and forth between two rotamers, suggesting an important role of $\mathrm{Na}^{+}$binding in stabilizing the eticlopride pose and the configuration of the $1 l e^{3.40}$ sub-pocket (Supplementary Fig. 2). Intriguingly, in the recently reported crystal structure of the serotonin $2 \mathrm{~A}$ receptor $\left(5-\mathrm{HT}_{2 \mathrm{~A}} \mathrm{R}\right)$ in complex with zotepine (PDB: $\left.6 \mathrm{~A} 94\right)^{20}$, the benzothiepin moiety of zotepine also occupies the $11 e^{3.40}$ sub-pocket, but this results in noticeable differences in the nearby residues compared to the $5-\mathrm{HT}_{2 \mathrm{~A}} \mathrm{R} /$ risperidone structure (PDB: 6A93) $)^{20}$. Specifically, the sidechain of the thermostabilizing mutation $5162^{3.39} \mathrm{~K}$, which occupies the $\mathrm{Na}^{+}$binding site, moves further away from $1 \mathrm{le} 163^{3.40}$ in the presence of the bound zotepine compared to that in the presence of risperidone. Such a difference indicates that zotepine and risperidone may have different sensitivities of $\mathrm{Na}^{+}$binding, which is consistent with the insensitivity of risperidone binding to $\mathrm{Na}^{+}$and the higher binding affinity of zotepine in the absence of $\mathrm{Na}^{+}$that we have previously observed at $\mathrm{D}_{2} \mathrm{R}^{19}$.

Together these findings along with our previously published data are consistent with our hypothesis that the binding of the ligands with different scaffolds stabilize distinct inactive conformations of $D_{2} R$ such that they are differentially sensitive to the presence of $\mathrm{Na}^{+}$, and there is likely an allosteric connection between the $\mathrm{Na}^{+}$binding site and the $1 \mathrm{e}^{3.40}$ sub-pocket.

\section{Plasticity of the ligand binding site in response to ligands with different scaffolds.}

On the extracellular side of the OBS, the space near Ser ${ }^{5.42}$ and $\operatorname{Ser}^{5.43}$ that accommodates the bulky substitutions of the benzamide rings of bound eticlopride and nemonapride in the $D_{3} R$ and $D_{4} R$ structures is not occupied by risperidone in $D_{2} R$. Furthermore, risperidone positions the aromatic cluster of TM6 and TM7 (Trp ${ }^{6.48}$, $\mathrm{Phe}^{6.51}, \mathrm{Phe}^{6.52}$, $\mathrm{His}^{6.55}$, and $\mathrm{Tyr}^{7.35}$ ) in $\mathrm{D}_{2} \mathrm{R}$ differently 
from its configurations in the $D_{3} R$ and $D_{4} R$ structures. These differences are likely associated with the inward and outward movements, respectively, of the extracellular portions of TM5 and TM6 in $D_{2} R$ relative to those in the $D_{3} R$ and $D_{4} R$ structures (Fig. 2a). To evaluate whether these conformational rearrangements are due to the minor divergence in these regions of the receptors or to the ligand binding site plasticity to accommodate ligands from different scaffolds, we compared the resulting conformations of $D_{2} R$ bound with risperidone or eticlopride. We observed the same trend of rearrangements of the transmembrane segments surrounding the OBS in the resulting receptor conformations from our $D_{2} R /$ risperidone and $D_{2} R /$ eticlopride simulations (Fig. $2 \mathrm{~b})$, i.e., an inward movement of TM6 and outward movement of TM5 in the presence of bound eticlopride (Fig. 2c,d). Without such movements in $D_{2}$ R/eticlopride, Ser193 5.42 and Ser194.43 would clash with the bound eticlopride (Fig. 2b). These findings further support our inference that these differences between the $D_{2} R$ and $D_{3} R$ inactive structures are largely due to the different scaffolds of the non-selective ligands bound.

\section{The extracellular loop 2 (EL2) of $D_{2} R /$ risperidone can spontaneously unwind.}

In addition to these differences in the transmembrane segments surrounding the OBS, there are also substantial differences in the configuration of EL2 in the $D_{2} R$ and $D_{3} R$ structures. EL2 (residues 173 to 186) between TM4 and TM5 is connected to TM3 via a disulfide bond formed between Cys $182^{\mathrm{EL} 2.50}$ (see Methods and Supplementary Fig. 3 for the indices of EL1 and EL2 residues) and Cys $107^{3.25}$. The conformation of EL2, the sequence of which is not conserved among aminergic GPCRs, is expected to be dynamic. Indeed, in the $D_{2} R /$ risperidone structure, the sidechains of residues $176^{\mathrm{EL} 2.40}, 178^{\mathrm{EL} 2.46}, 179^{\mathrm{EL} 2.47}$, and $180^{\mathrm{EL} 2.48}$, which are distal to the OBS were not solved, likely due to their dynamic nature. Curiously the portion of EL2 C-terminal to Cys $182^{\mathrm{EL} 2.50}$ (residues $182^{\mathrm{EL} 2.50}-186^{\mathrm{EL} 2.54}$ ), which forms the upper portion of the OBS that is in contact with ligand, is in a helical conformation in the $D_{2} R /$ risperidone structure. 
Strikingly, in our MD simulations, we found that this helical region showed a tendency to unwind (Supplementary Movie 1). The unwinding of EL2 involves a drastic rearrangement of the sidechain of Ile183 ${ }^{\mathrm{EL} 2.51}$, which must dissociate from a hydrophobic pocket formed by the sidechains of Val1113.29, Leu1704.60, Leu174 ${ }^{\mathrm{EL} 2.38}$, and Phe1895.38. This process is initiated by the loss of a hydrogen-bond (H-bond) interaction between the sidechain of Asp108.26 and the backbone amine group of Ile183 ${ }^{\mathrm{EL} 2.51}$ formed in the $\mathrm{D}_{2} \mathrm{R} /$ risperidone structure (Fig. $3 b$, step $\mathrm{i}$ ). When this interaction is broken, the orientation of residues $182^{\mathrm{EL} 2.50}-186^{\mathrm{EL} 2.54}$ deviates markedly from that of the crystal structure, losing its helical conformation (see below). Subsequently the sidechain of Ile $183^{\mathrm{EL} 2.51}$ rotates outwards and passes a small steric barrier of Gly173 ${ }^{\mathrm{EL} 2.37}$ (Fig. 3b, step ii), and in some trajectories makes a favorable hydrophobic interaction with the sidechain of Ala177 ${ }^{\mathrm{EL} 2.45}$. In a few long trajectories, Ile183 ${ }^{\mathrm{EL} 2.51}$ rotates further towards the extracellular vestibule where it can make favorable interactions with hydrophobic or aromatic residues from the $\mathrm{N}$ terminus, or the bound risperidone (Supplementary Movie 1). Consequently, residues $182^{\mathrm{EL} 2.50}-186^{\mathrm{EL} 2.54}$ are in a fully extended loop conformation while lle184 ${ }^{\mathrm{EL} 2.52}$ tilts under EL2 (Fig. 3b, step iii).

In the $D_{3} R$ structure, the aligned residue for Asp108 $8^{3.26}$ of $D_{2} R$ is conserved as Asp104 ${ }^{3.26}$; its sidechain forms an interaction not with Ile182 ${ }^{\mathrm{EL} 2.51}$ but rather with the sidechain of Asn173 ${ }^{\mathrm{EL} 2.39}$, which is also conserved in $D_{2} R$ as Asn175 ${ }^{\mathrm{EL} 2.39}$. In the $D_{4} R$, the aligned two residues (Asp1093.26 and $A s n 175^{\mathrm{EL} 2.39}$ ) are conserved as well, their sidechains are only $4.3 \AA$ away in the $\mathrm{D}_{4} \mathrm{R}$ structure, slightly larger than $3.2 \AA$ in the $D_{3} R$ structure. Even though these residues are conserved in $D_{2} R$, the interaction in $D_{3} R$ (and potentially in $D_{4} R$ ), between Asp ${ }^{3.26}-A s n^{E L 2.39}$, is not present in the $D_{2} R$ structure in which the aligned Asn175 ${ }^{\mathrm{EL} 2.39}$ faces lipid (Fig. 3a). However, in a few of our long $\mathrm{D}_{2} \mathrm{R}$ simulations, Asn175 ${ }^{\mathrm{EL} 2.39}$ gradually moves inwards and approaches Asp108 ${ }^{3.26}$ (Fig. 3b, step iv). At this point, the EL2 conformation of $D_{2} R$ is highly similar to that of $D_{3} R$ (Fig. 3c), suggesting that EL2 is dynamic and can exist in both conformations. 


\section{Both EL2 conformation and ligand scaffold affect the EL1 conformation.}

We have previously shown that the divergence in both the length and number of charged residues in EL1 among $D_{2} R, D_{3} R$, and $D_{4} R$ is responsible for the selectivity of more extended ligands ${ }^{21,22}$. Another striking difference in the $D_{2} R, D_{3} R$, and $D_{4} R$ structures is the position of the conserved $\operatorname{Trp}{ }^{\mathrm{EL} 1.50}$ in EL1. Trp100 ${ }^{\mathrm{EL} 1.50}$ is in a much more inward position in the $\mathrm{D}_{2} \mathrm{R}$ structure, making a direct contact with the bound risperidone (Fig. 4a), $\operatorname{Trp} 101^{\mathrm{EL} 1.50}$ in $\mathrm{D}_{4} \mathrm{R}$ interacts with the bound nemonapride that has an extended structure, whereas $\operatorname{Trp}^{\mathrm{EL} 1.50}$ in $\mathrm{D}_{3} R$ is not in contact with eticlopride (Fig. 4b). Thus, we asked whether these distinct positions of $\operatorname{Trp}^{\mathrm{EL} 1.50}$ are due to the divergence in EL1 among these receptors ${ }^{21}$ or due to the multiple inactive conformations that accommodate the binding of non-selective ligands of divergent scaffolds.

In the $D_{2} R /$ risperidone simulations, we found that when residues $182^{\mathrm{EL} 2.50}-186^{\mathrm{EL} 2.54}$ of $\mathrm{EL} 2$ are in a helical conformation and there is more room in the extracellular vestibule, the position of Trp100 10 L 1.50 is flexible and can adopt several positions and orientations (Fig. 4c,e,f). In the $D_{2}$ R/eticlopride simulations, when EL2 is helical, $\operatorname{Trp} 100^{\mathrm{EL} 1.50}$, which cannot interact with eticlopride, shows more flexibility and can move to a similar position like that of Trp96 ${ }^{\mathrm{EL} 1.50}$ in the $D_{3} R$ structure (Supplementary Fig. 4 and Supplementary Movie 2). Interestingly, in this position, the conformation of $\operatorname{Trp}^{\mathrm{EL} 1.50}$ can be stabilized by the disulfide bond of EL2 ${ }^{23}$ as shown in Supplementary Movie 2 or by interaction with the $\mathrm{N}$ terminus, which was truncated in the receptor used for the crystal structure. In the $D_{2} R /$ spiperone simulations, the phenyl substitution on the triazaspiro[4.5]decane moiety protrudes towards the interface between TM2 and TM3, and contacts Trp100 $10 \mathrm{~L} 1.50$, which is flexible as well but can adopt a position that is even further away from the OBS than that of $\operatorname{Trp} 96^{\mathrm{EL} 1.50}$ in the $\mathrm{D}_{3} \mathrm{R}$ structure (Supplementary Fig. 4).

In contrast, when EL2 is in an extended conformation like that in $D_{3} R$, it restricts the flexibility of $\operatorname{Trp} 100^{\mathrm{EL} 1.50}$ (Supplementary Movie 3). This trend is consistent with the $\mathrm{D}_{3} \mathrm{R} /$ eticlopride simulations in which we do not observe any significant rearrangement of Trp96 ${ }^{\mathrm{EL} 1.50}$ (Fig. 4d,e,f). 
Thus, we infer that the distinct conformation of $\operatorname{Trp} 100^{\mathrm{EL} 1.50}$ in the $\mathrm{D}_{2} \mathrm{R}$ structure is a combined effect of the helical EL2 conformation and the favored interaction that Trp100 ${ }^{\mathrm{EL} 1.50}$ can form with bound risperidone in the crystal structure, the latter of which however, has a minimal influence on the binding affinity of risperidone ${ }^{10}$, consistent with the unstable interaction between risperidone and $\operatorname{Trp} 100^{\mathrm{EL} 1.50}$ in our simulations (Fig. 4, Supplementary Movie 2). The mutation of this residue to alanine, leucine or phenylalanine did, however, cause substantial increases in both the association and dissociation rate of risperidone ${ }^{10}$. Thus, it appears that the conformation of EL2 influences the dynamics of $\operatorname{Trp} 100^{\mathrm{EL} 1.50}$, which in turn controls ligand access and egress to and from the OBS. Both the dissociation and association rates of $D_{2} R$ antagonists used as antipsychotics have been proposed to determine their propensity to cause extrapyramidal sideeffects and hyperprolactinaemia ${ }^{24}$. Understanding the relationship between the distinct inactive $D_{2} R$ conformations stabilized by different antagonist scaffolds and these kinetic parameters will likely be important to facilitate the design of $D_{2} R$ antagonists with an optimal kinetic profile that minimizes the risk of side effects.

\section{EL2 and EL1 dynamics.}

Next, we evaluated the tendency of the EL2 helix to unwind in each of the simulated $D_{2} R$ complexes, by measuring the stability of the backbone $\mathrm{H}$-bond between $\| \mathrm{e} 183^{\mathrm{EL} 2.51}$ and Asn186 ${ }^{\mathrm{EL} 2.54}$, a key stabilizing force of the helix (Fig. 5a). When we plotted the Ile183 ${ }^{\mathrm{EL} 2.51}$ Asn186 ${ }^{\mathrm{EL} 2.54}$ distance against the Asp108 ${ }^{3.26}$-Ile183 ${ }^{\mathrm{EL} 2.51}$ distance for each $\mathrm{D}_{2} \mathrm{R}$ complex (Fig. 5b), we found the loss of the Asp1083.26-Ile183 ${ }^{\mathrm{EL} 2.51}$ interaction increases the probability of breaking the Ile183 ${ }^{\mathrm{EL} 2.51}$-Asn186 ${ }^{\mathrm{EL} 2.54} \mathrm{H}$-bond, i.e., the unwinding of EL2. Interestingly, in all our simulated $D_{2} R$ complexes, EL2 has a clear tendency to unwind, regardless of the scaffold of the bound ligand (Fig. 5c,d, Supplementary Movies 1 and 3). Note in the $D_{3} R /$ eticlopride simulations, the aligned residues Ser182 $2^{\mathrm{EL} 2.51}$ and Asn185 ${ }^{\mathrm{EL} 2.54}$ does not form such a H-bond, and EL2 is always in an extended conformation (Fig. 5b-d). This trend of EL2 to transition towards the extended 
conformation is also present in our simulations of $D_{2} R$ in complex with a partial agonist, aripiprazole, whereas EL2 in the $D_{3} R$ complexes with partial agonists (R22 and S22) remains in the extended conformation (Supplementary Table 1 and Supplementary Fig. 5). Interestingly, Asp $104^{3.26}$ and Ser182 ${ }^{\mathrm{EL} 2.51}$ can move into interacting range in the $D_{3} R /$ eticlopride simulations, and the Ser182 ${ }^{\mathrm{EL} 2.51}$-Asn185 ${ }^{\mathrm{EL} 2.54}$ interaction can sporadically form in the $\mathrm{D}_{3} \mathrm{R} / \mathrm{R} 22$ simulations both raise a possibility that the extended conformation of $D_{3} R$ EL2 may transition to a helical conformation. In addition, while differences in the probabilities of unwinding for each $D_{2} R$ complex in Fig. 5 were not drastic, this may be a result of insufficient sampling and cannot exclude the possibility that different ligands may favor particular conformational equilibrium of EL2.

In the fully extended EL2 conformation in which Ile183 ${ }^{\mathrm{EL} 2.51}$ rotates to face the extracellular vestibule, Ile183 ${ }^{\mathrm{EL} 2.51}$ makes a direct contact with the bound risperidone, whereas $\operatorname{Trp} 100^{\mathrm{EL} 1.50}$ loses its interaction with the ligand entirely (Supplementary Movie 1). Nevertheless, risperidone retains all other contacts in the OBS. In the recently reported $5-\mathrm{HT}_{2 \mathrm{~A}} \mathrm{R} /$ risperidone structure (PDB: $6 A 93)^{20}$, risperidone has a very similar pose in the OBS as that in the $D_{2} R$ structure, occupying the $\mathrm{Il}^{3.40}$ sub-pocket as well. However, on the extracellular side of the OBS, EL2 in the 5$\mathrm{HT}_{2 \mathrm{~A}} \mathrm{R} /$ risperidone complex is in an extended conformation and the EL2 residue Leu228 ${ }^{\mathrm{EL} 2.51}$ contacting risperidone aligns to Ile $183^{\mathrm{EL} 2.51}$ of $\mathrm{D}_{2} \mathrm{R}$, whereas the conserved $\operatorname{Trp} 141^{\mathrm{EL} 1.50}$ does not interact with risperidone in the $5-\mathrm{HT}_{2 \mathrm{~A}} \mathrm{R}$. It is tempting to speculate the $\mathrm{EL} 2$ and $\mathrm{EL} 1$ dynamics we observe in the $D_{2} R /$ risperidone simulations represents a more comprehensive picture, as the divergent interactions shown in the extracellular loops of the $5-\mathrm{HT}_{2 \mathrm{~A}} \mathrm{R} /$ risperidone and $D_{2} R /$ risperidone structures may not result from differences in recognizing the ligand but rather two different static snapshots due a variety of differences in the crystallographic conditions (Note risperidone has similarly high affinities for both $D_{2} R$ and $5 H T_{2 A} R^{10,20}$ ). Interestingly, in one of our long $\mathrm{MD}$ trajectories of the $\mathrm{D}_{2} \mathrm{R} /$ risperidone complex, EL2 evolved into a conformation that has a helical N-terminal portion and an extended C-terminal portion (Supplementary Movie 4 and Supplementary Fig. 6). This conformation is not observed in either of the $D_{2} R /$ risperidone and 
$D_{3} R /$ eticlopride structures but is similar to that of the $5-H_{2} \mathrm{~A} /$ risperidone structure, further demonstrating the dynamics of this loop region (Supplementary Fig. 6).

Thus, the plasticity of the OBS and the dynamics of the extracellular loops appear to be two associated modules in ligand recognition. To the extent of our simulations, we did not detect strong ligand-dependent bias in the EL2 dynamics as we did for the OBS. However, when EL2 is helical, the EL1 dynamics are sensitive to the bound ligand (compare Fig. 4 and Supplementary Fig. 4); when EL2 is extended, it restricts the EL1 dynamics (Fig. 4). 


\section{DISCUSSION}

Our results highlight unappreciated conformational complexity for the inactive state of GPCRs and suggest that the risperidone bound $D_{2} R$ structure represents only one of a number of possible inactive conformations. Critically, this conformation is incompatible with the binding of other high affinity $D_{2} R$ ligands such as eticlopride. While distinct conformational states responsible for functional selectivity have garnered great attention, the potential existence of divergent inactive conformations is of critical importance for high-throughput virtual screening campaigns, as important hits (such as eticlopride) would be missed by focusing on a single antagonist bound state captured in a crystal structure with risperidone, an antagonist with a different scaffold. Such conformations may also confer differences in binding on and off rate or inverse agonist efficacy ${ }^{25}$, that in turn may confer distinct physiological or therapeutic effects. Furthermore, rational lead optimization requires rigorous physical description of molecular recognition ${ }^{26}$, which depends on adequate understanding of the conformational boundary and flexibility of the targeted state.

Previously, using the substituted-cysteine accessibility method (SCAM) in $D_{2} R^{27,28}$, we found that $\mathrm{G} 173^{\mathrm{EL} 2.37} \mathrm{C}, \mathrm{N} 175^{\mathrm{EL} 2.39} \mathrm{C}$, and $\mathrm{I} 184^{\mathrm{EL} 2.52} \mathrm{C}$ were accessible to the $\mathrm{MTS}$ reagents and that this accessibility could be blocked by the bound antagonist sulpiride, consistent with their water accessibility and involvement in ligand binding and not with a static orientation facing lipid, whereas $\mathrm{A} 177^{\mathrm{EL} 2.45} \mathrm{C}$ and $\mathrm{I} 183^{\mathrm{EL} 2.51} \mathrm{C}$ were accessible but not protected by sulpiride. Curiously, in the $D_{2} R /$ risperidone structure, Ile184 ${ }^{\mathrm{EL} 2.52}$ is only marginally in contact with the ligand, Ile $183^{\mathrm{EL} 2.51}$ blocks the accessibility of Gly $173^{\mathrm{EL} 2.37}$ to the OBS and is itself buried in a hydrophobic pocket, whereas Asn175 ${ }^{\mathrm{EL} 2.49}$ faces lipid, where it would be much less reactive. In the $D_{3} R$ /eticlopride structure, lle183 ${ }^{\mathrm{EL} 2.52}$ is in close contact with the bound ligand, Ser182 ${ }^{\mathrm{EL} 2.51}$ faces the extracellular vestibule, whereas the sidechain of Asn173 ${ }^{\mathrm{EL} 2.39}$ is oriented towards the OBS. Thus, it appears that the accessibility pattern of EL2 revealed by previous SCAM studies in $D_{2} R$ are more consistent with the extended EL2 conformation revealed by the $D_{3} R$ /eticlopride but not with the static $D_{2} R /$ risperidone structure (Supplementary Fig. 7). Indeed, we observed spontaneous 
transitions from the C-terminal helical EL2 conformation to a C-terminal extended conformation in our $D_{2} R$ simulations, which suggests that the EL2 conformation of $D_{2} R$ exists in an ensemble of structured and unwound conformations, with substantial occupation of the configuration found in $D_{3} R$. Such dynamics of EL2 would explain why the binding of non-selective ligands, such as risperidone or eticlopride, can result in drastically different conformations between the $D_{2} R$ and $D_{3} R$ structures near EL2, which are not related to the divergence of the receptors. Thus, the $D_{2} R$ EL2 appears to have quite dramatic dynamics that are not captured by the crystal structure.

In marked contrast to the obvious trend toward unwinding of EL2 in all our simulated $D_{2} R$ complexes, in our recent simulations of MhsT, a transporter protein with a region found by crystallography to alternate between helical and unwound conformations ${ }^{29}$, we failed to observe any spontaneous unwinding over a similar simulation timescale (with the longest simulations being $\sim 5-6 \mu \mathrm{s}$ ) when the region was started from the helical conformation ${ }^{30,31}$. This suggests that the C-terminal helical conformation of EL2 represents a higher energy state than the extended conformation, which allows for observation of the transitions in a simulation timescale not usually adequate to sample folding/unfolding events ${ }^{32}$.

Taken together, our findings reveal that both the plasticity of the transmembrane domain in accommodating different scaffolds and the dynamics of EL2 and EL1 are important considerations in RDD targeting the inactive conformation of $D_{2} R$. More extensive simulations and additional crystal structures bound with ligands from different scaffolds will help to fully reveal the correlations between these two modules. 


\section{METHODS}

\section{Residue indices in EL1 and EL2}

Based on a systematic analysis of aminergic receptors, we found a Trp in the middle of EL1 and the disulfide-bonded Cys in the middle of EL2 are the most conserved residues in each segment, and defined their residue indices as EL1.50 and EL2.50, respectively ${ }^{5}$, In this study, for the convenience of comparisons among $D_{2} R, D_{3} R$, and $D_{4} R$, and $5-H T_{2 A} R$, based on the alignments of EL1 And EL2 shown in Supplementary Fig. 3, we index the EL1 and EL2 residues of each receptor in the same way as the Ballesteros-Weinstein numbering, e.g., the residues before and after the EL2.50 are EL2.49 and EL2.51, respectively. Note the indices for the shorter sequences are not be consecutive, given the gaps in the alignment.

\section{Molecular modeling and docking}

The $D_{2} R$ models in this study are based on the corrected crystal structure of $D_{2} R$ bound to risperidone (PDB: 6CM4) ${ }^{10}$. We omitted T4 Lysozyme fused into intracellular loop 3. Three thermostabilizing mutations (Ile $122^{3.40} \mathrm{~A}, \mathrm{~L} 375^{6.37} \mathrm{~A}$, and $\mathrm{L} 379^{6.41} \mathrm{~A}$ ) were reverted to their WT residues. The missing $\mathrm{N}$ terminus in the crystal structure was built de novo using Rosetta ${ }^{33}$, and then integrated with the rest of the $D_{2} R$ model using Modeller ${ }^{34}$. Using Modeller, we also extended two helical turns at the TM5 $\mathrm{C}$ terminus and threes residues at the TM6 N terminus of the structure and connected these two ends with a 9 Gly loop, similar to our experimentally validated treatment of D3R models ${ }^{35}$. The position of the $\mathrm{Na}^{+}$bound in the canonical $\mathrm{Na}^{+}$binding site near the negatively charged $\mathrm{Asp}^{2.50}$ was acquired by superimposing the $\mathrm{Na}^{+}$bound structure of adenosine $A_{2 A}$ receptor ${ }^{36}$ to our $D_{2} R$ models.

The binding poses of risperidone and eticlopride were taken according to their poses in the $D_{2} R^{10}$ and $D_{3} R^{9}$ structures, respectively. Docking of spiperone in our D2R model was performed using the induced-fit docking (IFD) protocol $^{37}$ in the Schrodinger software (release 2017-2; 
Schrodinger, LLC: New York NY). Based on our hypothesis regarding the role of the lle ${ }^{3.40}$ subpocket in the $\mathrm{Na}^{+}$sensitivity (see text), from the resulting poses of IFD, we choose the spiperone pose with the F-substitution on the butyrophenone ring occupying the $11 e^{3.40}$ sub-pocket. Note that in risperidone and spiperone the F-substitutions have similar distances to the protonated $\mathrm{N}$ atoms that interact with $\mathrm{Asp}^{3.32}$ (measured by the number of carbon atoms between them, Supplementary Fig. 1).

\section{Molecular dynamics (MD) simulations}

MD simulations of the $D_{2} R$ and $D_{3} R$ complexes were performed in the explicit water and 1palmitoyl-2-oleoylphosphatidylcholine (POPC) lipid bilayer environment using Desmond MD System (version 4.5; D. E. Shaw Research, New York, NY) with either the OPLS3e force field ${ }^{38}$ or the CHARMM36 force field ${ }^{39-42}$ and TIP3P water model. For CHARMM36 runs, the eticlopride parameters were obtained through the GAAMP server ${ }^{43}$, with the initial force field based on CGenFF assigned by ParamChem ${ }^{44}$. The system charges were neutralized, and $150 \mathrm{mM} \mathrm{NaCl}$ was added. Each system was first minimized and then equilibrated with restraints on the ligand heavy atoms and protein backbone atoms, followed by production runs in an isothermal-isobaric (NPT) ensemble at $310 \mathrm{~K}$ and 1 atom with all atoms unrestrained, as described previously ${ }^{19,35}$. We used Langevin constant pressure and temperature dynamical system ${ }^{45}$ to maintain the pressure and the temperature, on an anisotropic flexible periodic cell with a constant-ratio constraint applied on the lipid bilayer in the $X-Y$ plane. For each condition, we collected multiple trajectories, the aggregated simulation length is $\sim 340 \mu$ s (Table S1).

While the majority of our $D_{2} R$ simulations in this study used the OPLS3 force field, to compare with the $D_{3} R$ simulations using CHARMM36 that have been continued from the previously reported shorter trajectories ${ }^{19,35}$, we carried out the $D_{2} R /$ eticlopride simulations using both the OPLS3 and CHARMM36 force fields (see Table S1). We did not observe significant differences and pooled their results together for the analysis. 


\section{Conformational analysis}

Distances and dihedral angles of MD simulation results were calculated with MDTraj (version 1.8.2 $)^{46}$ in combination with in-house Python scripts.

To characterize the structural changes in the receptor upon ligand binding, we quantified differences of structural elements between the $D_{2} R$ /eticlopride and $D_{2} R /$ risperidone conditions (using last 600 ns from a representative trajectory for each condition), by applying the previously described pairwise interaction analyzer for GPCR (PIA-GPCR) ${ }^{35}$. The subsegments on the extracellular side of $D_{2} R$ were defined as following: TM1e (the extracellular subsegment (e) of TM1, residues 31-38), TM2e (residues 92-96), TM3e (residues 104-113), TM4e (residues 166172), TM5e (residues 187-195), TM6e (residues 364-369), and TM7e (residues 376-382).

For the PIA-GPCR analysis and the distance analysis in Fig. 4, we used the set of ligand binding residues previously identified by our systematic analysis of GPCR structures. Specifically, for $D_{2} R$, they are residues $91,94,95,100,110,111,114,115,118,119,122,167,184,189,190$, $193,194,197,198,353,357,360,361,364,365,367,368,376,379,380,383,384$, 386, and 387 ; for $D_{3} R$, they are residues $86,89,90,96,106,107,110,111,114,115,118,165,183,188$, $189,192,193,196,197,338,342,345,346,349,350,352,353,362,365,366,369,370,372$, and 373.

\section{Markov State Model (MSM) analysis}

The MSM analysis was performed using the pyEMMA program (version 2.5.5) ${ }^{47}$. To characterize the dynamics of EL2 of $D_{2} R$, specifically the transitions between helical and extended conformations of its C-terminal portion, we focused on a key hydrogen bond formed in the helical conformation between the backbone carbonyl group of lle183 and the backbone amine group of Asn186. Thus, for each of the simulated conditions, the distance of lle183-Asn186 (Ser182- 
Asn185 in $D_{3} R$ ) was used as an input feature for the MSM analysis. We discretized this feature into two clusters - distances below and above $4 \AA$ (i.e. EL2 forming a helical conformation and unwinding). Implied relaxation timescale (ITS $)^{48}$ for the transition between these clusters was obtained as a function of various lag times. Convergences of ITS for the MSMs for all conditions was achieved at a lag time of 300 ns (Supplementary Fig. 8), which we further used to estimate Bayesian Markov models with 500 transition matrix samples ${ }^{49}$. The maximum likelihood transition matrix was used to calculate the transition and equilibrium probabilities $(\pi)$ shown in Fig. 5 and Supplementary Fig. 5.

\section{$\left[{ }^{3} \mathrm{H}\right]$ spiperone binding assay.}

For saturating binding assays cell membranes (SNAP-D $2 \mathrm{~s}-\mathrm{Flpln} \mathrm{CHO}, 2.5 \mu \mathrm{g}$ ) were incubated with varying concentrations of $\left[{ }^{3} \mathrm{H}\right]$ spiperone and $10 \mu \mathrm{M}$ haloperidol as a non-specific control, in binding buffer (20 mM HEPES, $100 \mathrm{mM} \mathrm{NaCl}, 6 \mathrm{mM} \mathrm{MgCl} 2,1 \mathrm{mM} \mathrm{EGTA}$, and 1mM EDTA, pH 7.4) to a final volume of $200 \mu \mathrm{L}$ and were incubated at $37^{\circ} \mathrm{C}$ for $3 \mathrm{~h}$. For competition binding assays cell membranes $\left(\right.$ SNAP- $\left.{ }_{2 s}-F I p l n ~ C H O, 2.5 \mu g\right)$ were incubated with varying concentrations of test compound in binding buffer containing $0.2 \mathrm{nM}$ of $\left[{ }^{3} \mathrm{H}\right]$ spiperone to a final volume of $200 \mu \mathrm{L}$ and were incubated at $37^{\circ} \mathrm{C}$ for $3 \mathrm{~h}$. Binding was terminated by fast-flow filtration using a Uniplate 96-well harvester (PerkinEImer) followed by five washes with ice-cold $0.9 \% \mathrm{NaCl}$. Bound radioactivity was measured in a MicroBeta2 LumiJET MicroBeta counter (PerkinElmer).

\section{Data analysis}

The concentration of ligand that inhibited half of the $\left[{ }^{3} \mathrm{H}\right]$ spiperone binding $\left(\mathrm{IC}_{50}\right)$ was determined using the following equation:

$$
Y=\frac{\text { Bottom }+(\text { Top }- \text { Bottom })}{1+10^{\left(X-\text { LogIC }_{50}\right) n_{H}}}
$$


bioRxiv preprint doi: https://doi.org/10.1101/640870; this version posted May 17, 2019. The copyright holder for this preprint (which was not certified by peer review) is the author/funder. This article is a US Government work. It is not subject to copyright under 17 USC 105 and is also made available for use under a CCO license.

Where $\mathrm{Y}$ denotes the percentage specific binding, Top and Bottom denote the maximal and minimal asymptotes, respectively, $I_{50}$ denotes the $X$-value when the response is midway between Bottom and Top, and $n \mathrm{H}$ denotes the Hill slope factor. $\mathrm{IC}_{50}$ values obtained from the inhibition curves were converted to $K_{\mathrm{i}}$ values using the Cheng and Prusoff equation. 


\section{Acknowledgements}

Support for this research was provided by the National Institute on Drug Abuse-Intramural Research Program, Z1A DA000606-03 (L.S.), NIH grant MH54137 (J.A.J.) and National Health and Medical Research Council (NHMRC) Project Grant APP1049564 (J.R.L)

\section{Author Contributions}

J.R.L., J.A.J., and L.S. designed the studies. A.M.A. R.K.V., and L.S. performed computational modeling, simulations, and analysis. J.R.L. and H.D.L. performed the binding assay. J.R.L., J.A.J., and L.S. wrote the manuscript, with contributions from all the authors. Jackie Glenn is thanked for technical support in generating membrane preparations.

\section{Competing financial interests}

The authors declare no competing financial interests. 


\section{TABLES}

Table 1. The effect of mutations on the binding affinities of selected $D_{2} R$ ligands. The affinities of $\left[{ }^{3} \mathrm{H}\right]$ spiperone were determined in saturation experiments at WT or mutant SNAP-tagged D2SRs stably expressed in FlpIN CHO cells. Binding affinity values for aripiprazole and risperidone were obtained in competition binding experiments. Values are expressed as mean \pm S.E.M. from three separate experiments. ${ }^{*} \mathrm{P}<0.05$, significantly different from the wild-type receptor determined by a one-way ANOVA, Dunnett post-hoc test.

\begin{tabular}{|c|c|c|c|c|}
\hline & $\begin{array}{l}{\left[{ }^{3} \mathrm{H}\right] \text { spiperone }} \\
\text { saturation bir }\end{array}$ & ding & $\begin{array}{l}{\left[{ }^{3} \mathrm{H}\right] \text { spiperone }} \\
\text { competition binding }\end{array}$ & \\
\hline $\begin{array}{l}\text { SNAP- } \\
\mathrm{D}_{2 \mathrm{~S}} \mathrm{R}\end{array}$ & $\begin{array}{c}p K_{d} \\
\left(K_{d}, n M\right)\end{array}$ & $\begin{array}{c}\mathrm{B}_{\max } \\
(\text { pmol.mg-1) }\end{array}$ & $\begin{array}{l}\text { Risperidone } \\
\mathrm{p} K_{\mathrm{i}}\left(K_{\mathrm{i}}, \mathrm{nM}\right)\end{array}$ & $\begin{array}{l}\text { Eticlopride } \\
\mathrm{p} K_{\mathrm{i}}\left(K_{\mathrm{i}}, \mathrm{nM}\right)\end{array}$ \\
\hline WT & $\begin{array}{c}9.72 \pm 0.11 \\
(0.19)\end{array}$ & $7.95 \pm 1.63$ & $8.55 \pm 0.20(2.8)$ & $\begin{array}{c}9.84 \pm 0.17 \\
(0.14)\end{array}$ \\
\hline WT $-\mathrm{Na}^{+}$ & $\begin{array}{c}9.70 \pm 0.11 \\
(0.20)\end{array}$ & $6.39 \pm 1.04$ & $8.96 \pm 0.04(1.1)$ & - \\
\hline $1122^{3.40} \mathrm{~A}$ & $\begin{array}{c}9.74 \pm 0.15 \\
(0.18)\end{array}$ & $4.37 \pm 0.92$ & $8.10 \pm 0.03(7.9)$ & $\begin{array}{c}10.33 \pm 0.04^{*} \\
(0.04)\end{array}$ \\
\hline $1122^{3.40} \mathrm{~W}$ & $\begin{array}{c}8.95 \pm 0.08^{*} \\
(1.15)\end{array}$ & $2.61 \pm 0.50$ & $7.43 \pm 0.12^{*}(37)$ & $\begin{array}{c}9.61 \pm 0.0 \\
(0.25)\end{array}$ \\
\hline
\end{tabular}




\section{REFERENCES}

1 Weis, W. I. \& Kobilka, B. K. The Molecular Basis of G Protein-Coupled Receptor Activation. Annu Rev Biochem 87, 897-919, doi:10.1146/annurev-biochem-060614-033910 (2018).

2 Venkatakrishnan, A. J. et al. Molecular signatures of G-protein-coupled receptors. Nature 494, 185-194, doi:10.1038/nature11896 (2013).

3 Latorraca, N. R., Venkatakrishnan, A. J. \& Dror, R. O. GPCR Dynamics: Structures in Motion. Chem Rev 117, 139-155, doi:10.1021/acs.chemrev.6b00177 (2017).

4 Congreve, M., Dias, J. M. \& Marshall, F. H. Structure-based drug design for G proteincoupled receptors. Prog Med Chem 53, 1-63, doi:10.1016/B978-0-444-63380-4.00001-9 (2014).

5 Michino, M. et al. What can crystal structures of aminergic receptors tell us about designing subtype-selective ligands? Pharmacol Rev 67, 198-213, doi:10.1124/pr.114.009944 (2015).

6 McCorvy, J. D. et al. Structure-inspired design of beta-arrestin-biased ligands for aminergic GPCRs. Nat Chem Biol 14, 126-134, doi:10.1038/nchembio.2527 (2018).

7 Free, R. B. et al. Discovery and characterization of a G protein-biased agonist that inhibits beta-arrestin recruitment to the D2 dopamine receptor. Mol Pharmacol 86, 96-105, doi:10.1124/mol.113.090563 (2014).

8 Manglik, A. et al. Structure-based discovery of opioid analgesics with reduced side effects. Nature 537, 185-190, doi:10.1038/nature19112 (2016).

9 Chien, E. Y. et al. Structure of the human dopamine D3 receptor in complex with a D2/D3 selective antagonist. Science 330, 1091-1095, doi:10.1126/science.1197410 (2010).

10 Wang, S. et al. Structure of the D2 dopamine receptor bound to the atypical antipsychotic drug risperidone. Nature 555, 269-273, doi:10.1038/nature25758 (2018).

11 Sibley, D. R. \& Shi, L. A new era of rationally designed antipsychotics. Nature 555, 170172, doi:10.1038/d41586-018-02328-z (2018). 
12 Wang, S. et al. D4 dopamine receptor high-resolution structures enable the discovery of selective agonists. Science 358, 381-386, doi:10.1126/science.aan5468 (2017).

13 Ballesteros, J. \& Weinstein, H. Integrated methods for the construction of threedimensional models of structure-function relations in $\mathrm{G}$ protein-coupled receptors. Methods in Neurosciences 25, 366-428 (1995).

14 Silvestre, J. S. \& Prous, J. Research on adverse drug events. I. Muscarinic M3 receptor binding affinity could predict the risk of antipsychotics to induce type 2 diabetes. Methods Find Exp Clin Pharmacol 27, 289-304, doi:10.1358/mf.2005.27.5.908643 (2005).

15 Hirose, T. \& Kikuchi, T. Aripiprazole, a novel antipsychotic agent: dopamine D2 receptor partial agonist. J Med Invest 52 Suppl, 284-290 (2005).

16 Roth, B. L., Lopez, E., Patel, S. \& Kroeze, W. K. The Multiplicity of Serotonin Receptors: Uselessly Diverse Molecules or an Embarrassment of Riches? The Neuroscientist 6, 252262, doi:10.1177/107385840000600408 (2000).

17 Rasmussen, S. G. et al. Structure of a nanobody-stabilized active state of the beta(2) adrenoceptor. Nature 469, 175-180, doi:10.1038/nature09648 (2011).

18 Herenbrink, C. K. et al. Molecular determinants of the intrinsic efficacy of the antipsychotic aripiprazole. (Submitted).

19 Michino, M., Free, R. B., Doyle, T. B., Sibley, D. R. \& Shi, L. Structural basis for Na(+)sensitivity in dopamine D2 and D3 receptors. Chem Commun (Camb) 51, 8618-8621, doi:10.1039/c5cc02204e (2015).

20 Kimura, K. T. et al. Structures of the 5-HT2A receptor in complex with the antipsychotics risperidone and zotepine. Nat Struct Mol Biol 26, 121-128, doi:10.1038/s41594-018-0180z (2019).

21 Michino, M. et al. A single glycine in extracellular loop 1 is the critical determinant for pharmacological specificity of dopamine D2 and D3 receptors. Mol Pharmacol 84, 854864, doi:10.1124/mol.113.087833 (2013). 
22 Newman, A. H. et al. Molecular determinants of selectivity and efficacy at the dopamine D3 receptor. J Med Chem 55, 6689-6699, doi:10.1021/jm300482h (2012).

23 loerger, T. R., Du, C. \& Linthicum, D. S. Conservation of cys-cys trp structural triads and their geometry in the protein domains of immunoglobulin superfamily members. $\mathrm{Mol}$ Immunol 36, 373-386 (1999).

24 Seeman, P. Clozapine, a fast-off-D2 antipsychotic. ACS Chem Neurosci 5, 24-29, doi:10.1021/cn400189s (2014).

25 Roberts, D. J. \& Strange, P. G. Mechanisms of inverse agonist action at D2 dopamine receptors. Br J Pharmacol 145, 34-42, doi:10.1038/sj.bjp.0706073 (2005).

26 Beuming, T. \& Shi, L. Editorial: Computer Aided Structure-based Lead Optimization. Curr Top Med Chem 17, 2575-2576, doi:10.2174/156802661723170808161306 (2017).

27 Javitch, J. A. et al. The fourth transmembrane segment of the dopamine D2 receptor: accessibility in the binding-site crevice and position in the transmembrane bundle. Biochemistry 39, 12190-12199 (2000).

28 Shi, L. \& Javitch, J. A. The second extracellular loop of the dopamine D2 receptor lines the binding-site crevice. Proc Natl Acad Sci $U$ S A 101, 440-445, doi:10.1073/pnas.2237265100 (2004).

29 Malinauskaite, L. et al. A mechanism for intracellular release of $\mathrm{Na}+$ by neurotransmitter/sodium symporters. Nat Struct Mol Biol 21, 1006-1012, doi:10.1038/nsmb.2894 (2014).

30 Stolzenberg, S. et al. The role of transmembrane segment 5 (TM5) in Na2 release and the conformational transition of neurotransmitter:sodium symporters toward the inward-open state. J Biol Chem 292, 7372-7384, doi:10.1074/jbc.M116.757153 (2017).

31 Abramyan, A. M., Quick, M., Xue, C., Javitch, J. A. \& Shi, L. Exploring Substrate Binding in the Extracellular Vestibule of MhsT by Atomistic Simulations and Markov Models. $J$ Chem Inf Model 58, 1244-1252, doi:10.1021/acs.jcim.8b00175 (2018). 
32 Piana, S., Lindorff-Larsen, K. \& Shaw, D. E. How robust are protein folding simulations with respect to force field parameterization? Biophys $J$ 100, L47-49, doi:10.1016/j.bpj.2011.03.051 (2011).

33 Bradley, P., Misura, K. M. \& Baker, D. Toward high-resolution de novo structure prediction for small proteins. Science 309, 1868-1871, doi:10.1126/science.1113801 (2005).

34 John, B. \& Sali, A. Comparative protein structure modeling by iterative alignment, model building and model assessment. Nucleic Acids Res 31, 3982-3992 (2003).

35 Michino, M. et al. Toward Understanding the Structural Basis of Partial Agonism at the Dopamine D3 Receptor. J Med Chem 60, 580-593, doi:10.1021/acs.jmedchem.6b01148 (2017).

36 Liu, W. et al. Structural basis for allosteric regulation of GPCRs by sodium ions. Science 337, 232-236, doi:10.1126/science.1219218 (2012).

37 Sherman, W., Day, T., Jacobson, M. P., Friesner, R. A. \& Farid, R. Novel procedure for modeling ligand/receptor induced fit effects. J Med Chem 49, 534-553, doi:10.1021/jm050540c (2006).

38 Roos, K. et al. OPLS3e: Extending Force Field Coverage for Drug-Like Small Molecules. J Chem Theory Comput 15, 1863-1874, doi:10.1021/acs.jctc.8b01026 (2019).

39 MacKerell, A. D. et al. All-Atom Empirical Potential for Molecular Modeling and Dynamics Studies of Proteinst. J Phys Chem B 102, 3586-3616, doi:10.1021/jp973084f (1998).

40 MacKerell, A. D., Jr., Feig, M. \& Brooks, C. L., 3rd. Improved treatment of the protein backbone in empirical force fields. J Am Chem Soc 126, 698-699 (2004).

41 Best, R. B. et al. Optimization of the additive CHARMM all-atom protein force field targeting improved sampling of the backbone phi, psi and side-chain chi(1) and chi(2) dihedral angles. J Chem Theory Comput 8, 3257-3273, doi:10.1021/ct300400x (2012).

42 Klauda, J. B. et al. Update of the CHARMM all-atom additive force field for lipids: validation on six lipid types. J Phys Chem B 114, 7830-7843, doi:10.1021/jp101759q (2010). 
43 Huang, L. \& Roux, B. Automated Force Field Parameterization for Non-Polarizable and Polarizable Atomic Models Based on Ab Initio Target Data. J Chem Theory Comput 9, doi:10.1021/ct4003477 (2013).

44 Vanommeslaeghe, K. et al. CHARMM general force field: A force field for drug-like molecules compatible with the CHARMM all-atom additive biological force fields. $J$ Comput Chem 31, 671-690, doi:10.1002/jcc.21367 (2010).

45 Feller, S. E., Zhang, Y., Pastor, R. W. \& Brooks, B. R. Constant pressure molecular dynamics simulation: The Langevin piston method. J Chem Phys 103, 4613-4621 (1995).

46 McGibbon, R. T. et al. MDTraj: A Modern Open Library for the Analysis of Molecular Dynamics Trajectories. Biophys J 109, 1528-1532, doi:10.1016/j.bpj.2015.08.015 (2015).

47 Scherer, M. K. et al. PyEMMA 2: A Software Package for Estimation, Validation, and Analysis of Markov Models. J Chem Theory Comput 11, 5525-5542, doi:10.1021/acs.jctc.5b00743 (2015).

48 Swope, W. C., Pitera, J. W. \& Suits, F. Describing Protein Folding Kinetics by Molecular Dynamics Simulations. 1. Theory†. The Journal of Physical Chemistry B 108, 6571-6581, doi:10.1021/jp037421y (2004).

49 Trendelkamp-Schroer, B. \& Noe, F. Efficient Bayesian estimation of Markov model transition matrices with given stationary distribution. J Chem Phys 138, 164113, doi:10.1063/1.4801325 (2013). 


\section{FIGURES AND FIGURE LEGENDS}

Figure 1. Divergent occupations of the $1 \mathrm{e}^{3.40}$ sub-pocket by non-selective ligands from different scaffolds. In the $D_{2} R$ structure (a), the F-substitution on the benzisoxazole ring of risperidone occupies the $\| \mathrm{e}^{3.40}$ sub-pocket enclosed by conserved $\| \mathrm{e}^{3.40}, \mathrm{Phe}^{5.47}$, and $\mathrm{Ph} \mathrm{e}^{6.44}$. The same viewing angle shows that in the $D_{3} R(\mathbf{b})$ and $D_{4} R(\mathbf{c})$ structures, Cys ${ }^{3.36}$ rotates to fill in the lle $e^{3.40}$ sub-pocket, and the substituted benzamides eticlopride and nemonapride cannot occupy the aligned sub-pockets. In our $\mathrm{D}_{2} \mathrm{R} /$ risperidone simulations (d), risperidone maintains its pose revealed by the crystal structure. In the $D_{2} R /$ spiperone simulations (e), the $11 e^{3.40}$ sub-pocket is similarly occupied as in $D_{2} R /$ risperidone. In the $D_{2} R /$ eticlopride simulations (f), the $11 e^{3.40}$ subpocket is collapsed as in the $D_{3} R(\mathbf{b})$ and $D_{4} R(\mathbf{c})$ structures (this trend is independent of the force field being used in the simulations).

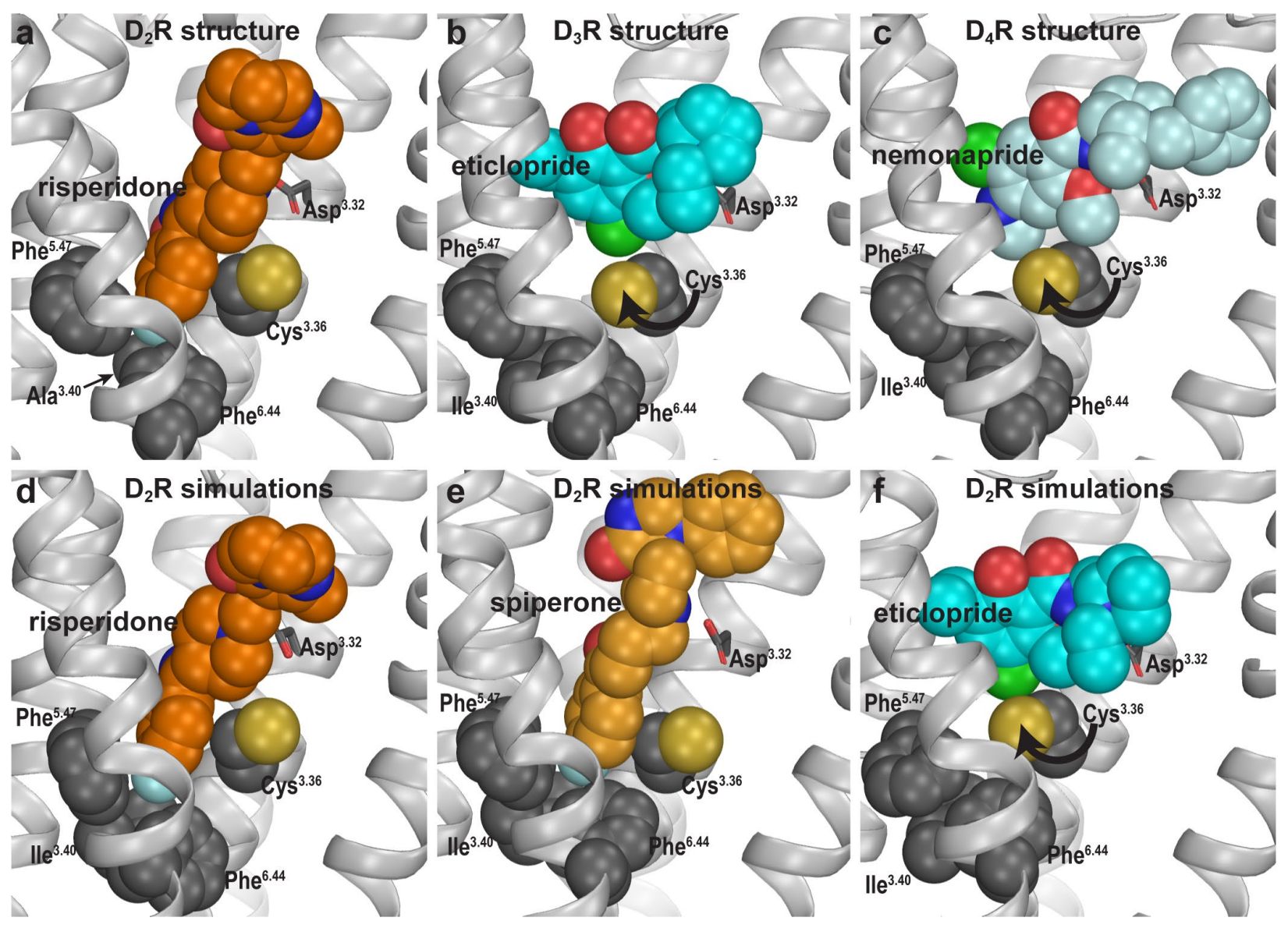


Figure 2. The different conformations in the extracellular vestibules of $D_{2} R, D_{3} R$, and $D_{4} R$ are likely due to binding of non-selective ligands from different scaffolds. (a) Superpositioning of $D_{2} R$, $D_{3} R$, and $D_{4} R$ structures shows that the binding of eticlopride (ETQ, cyan) in $D_{3} R$ and nemonapride (NEMO, pale cyan) in $D_{4} R$ result in outward and inward rearrangements of the extracellular portions of TM5 and TM6, respectively, compared to the binding of risperidone (RISP, orange) in $D_{2} R$. (b) superpositioning of representative frames of the $D_{2} R / E T Q$ and $D_{2} R / R I S P$ simulations shows a similarly trend of the outward and inward movements of TM5 and TM6, respectively, in the presence of the bound ETQ, even when the simulations were started from the $D_{2} R$ conformation stabilized by RISP. Note Ser $193^{5.42}$ and Ser $194^{5.43}$ would clash with the bound eticlopride if there was conformational adjustment. (c, d) PIA-GPCR analysis (see Methods) comparing the $D_{2} R / E T Q$ and $D_{2} R / R I S P$ conformations. The analysis of the pairwise-distance differences among the subsegments (c) indicates that TM6e moves inward (smaller distance to TM2e, dark red pixel), while TM5e moves outward (larger distances to TM7e, dark blue pixel) in the $D_{2} R / E T Q$ simulations. The analysis of pairwise-distance differences among the C $\alpha$ atoms of the ligand binding residues (d) indicates significant changes near residues Phe1895.38, Ser193 ${ }^{5.42}$, Asn367 6.58 , and 11 e368 6.59 (darker colored pixels). 


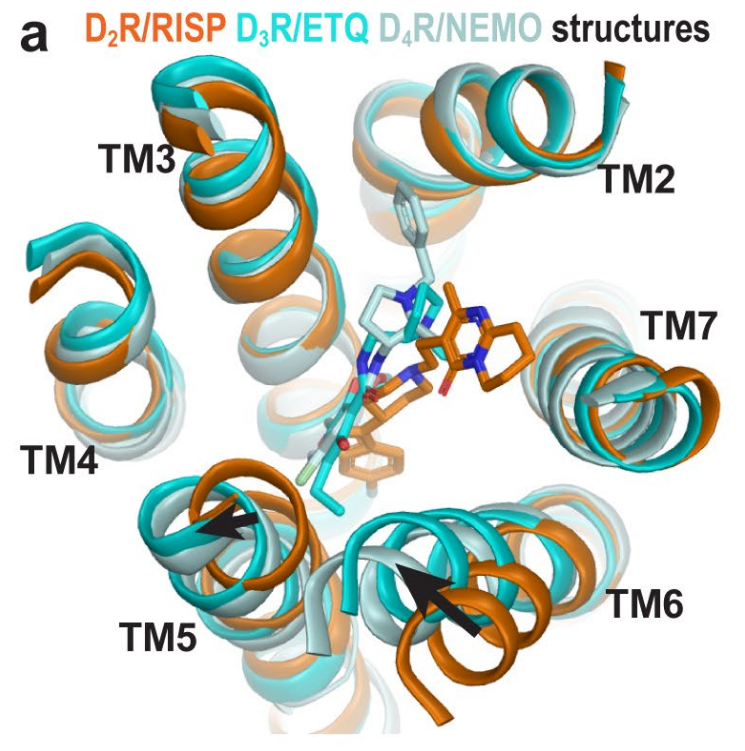

C

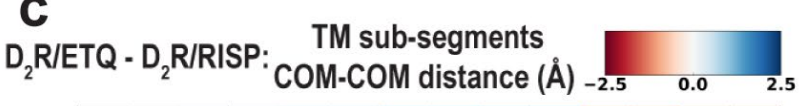

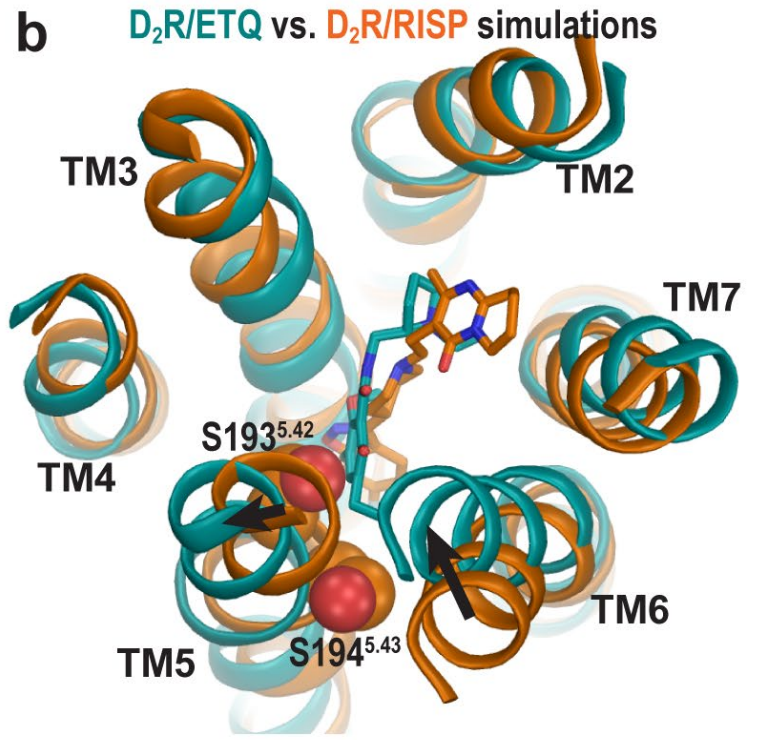

d

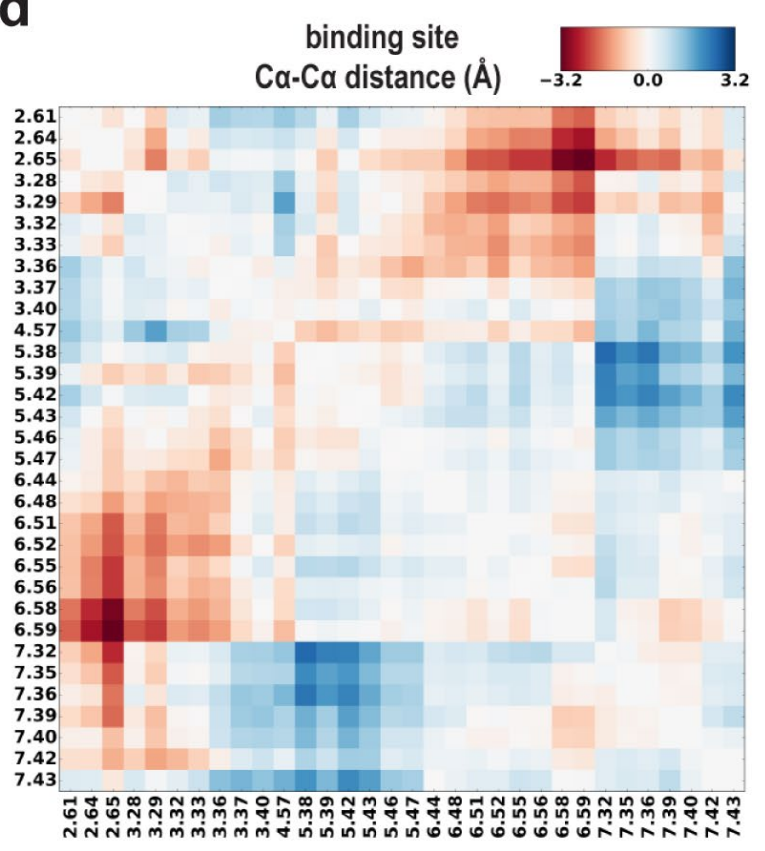


Figure 3. The helical region of EL2 of $D_{2} R$ can spontaneously unwind to an extended conformation similar to that of $D_{3} R$. (a) Residues $182^{\mathrm{EL} 2.50}-186^{\mathrm{EL} 2.54}$ in the $D_{2} R / R I S P$ structure are in a helical conformation. EL2 is connected to TM3 via a disulfide bond (Cys182 ${ }^{\mathrm{EL} 2.50}-\mathrm{Cys} 107^{3.25}$ ), while the backbone of Ile183 ${ }^{\mathrm{EL} 2.51}$ forms an interaction with Asp108 ${ }^{3.26}$ (magenta dotted line). (b) The key events in the EL2 unwinding pathway (for each step, a number of representative frames are shown): the ionic interaction between $A s p 108^{3.26}$ and Ile183 ${ }^{\mathrm{EL} 2.51}$ has to dissociate first (i), which allows the sidechain of lle183 to rotate towards lipids and pass through a minor barrier formed by Gly173 ${ }^{\mathrm{EL} 2.37}$ (ii); then the sidechain of Ile183 ${ }^{\mathrm{EL} 2.51}$ rotates towards the extracellular vestibule while that of Ile184 ${ }^{\mathrm{EL} 2.52}$ tilts under EL2 (iii); these changes allow Asn175 ${ }^{\mathrm{EL} 2.39}$ to move from facing lipid to facing the binding site (iv). The resulting conformation of EL2 of $D_{2} R$ is similar to that of $D_{3} R$ for all the aforementioned residues (c). In particular, Asn173 ${ }^{E L 2.39}$ of $D_{3} R$, which aligns to Asn $175^{\mathrm{EL} 2.39}$ of $\mathrm{D}_{2} \mathrm{R}$, forms an H-bond interaction with Asp104 ${ }^{3.26}$.

a $\quad D_{2} R / R I S P$

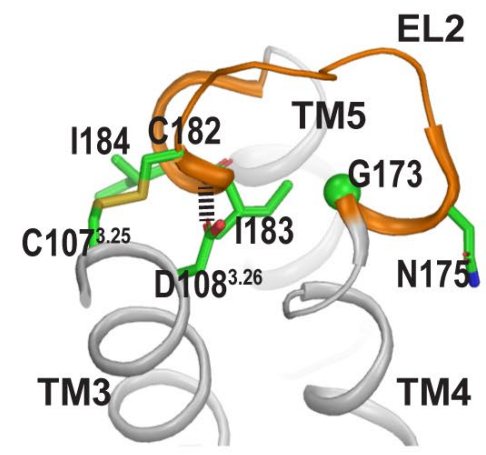

C $D_{3} R / E T Q$

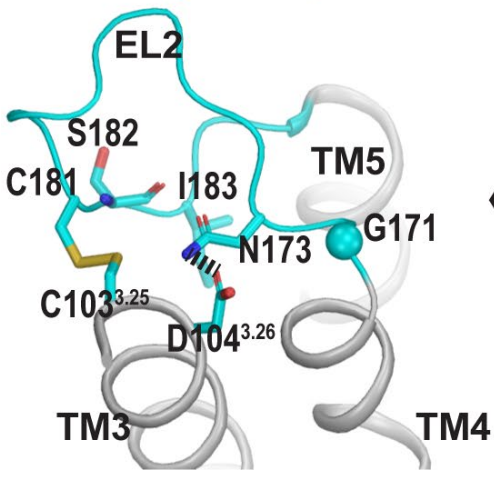

b

EL2 unwinding pathway
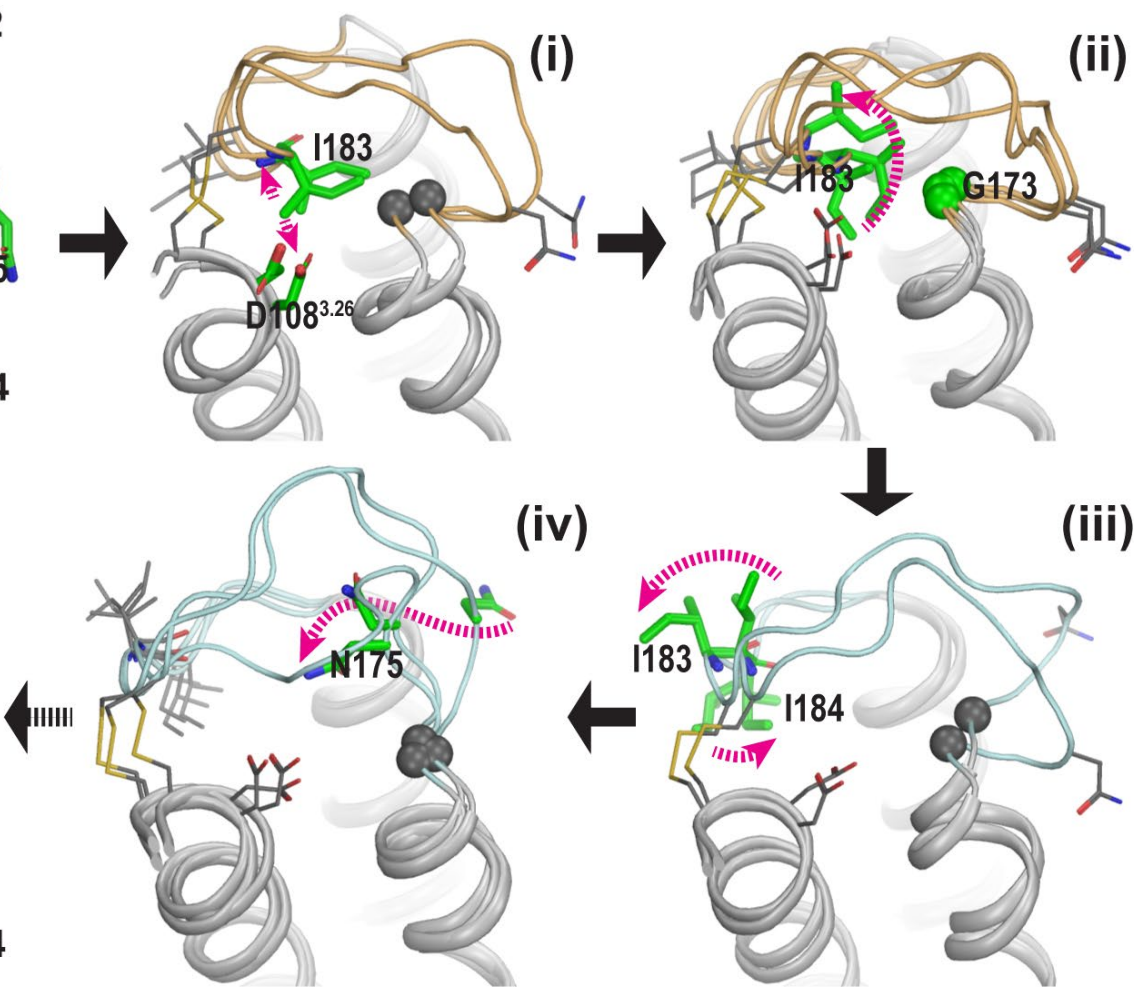

(iv)

(iii)

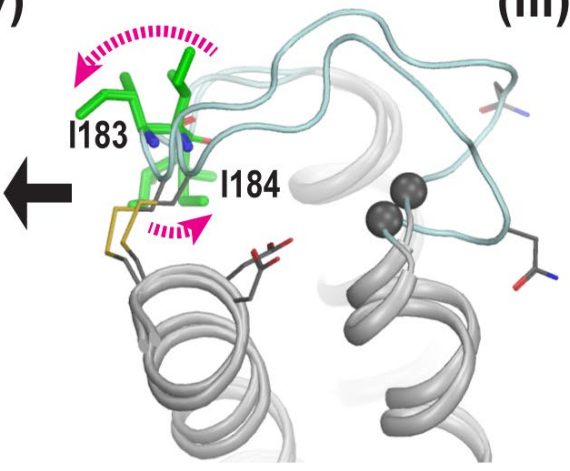


Figure 4. The EL2 conformation affects the EL1 conformation. Divergent EL1-EL2 interfaces among the $D_{2} R(\mathbf{a}), D_{3} R$, and $D_{4} R(\mathbf{b})$ structures. In the $D_{2} R$ structure, the Trp100 ${ }^{E L 1.50}$ in EL1 only forms a weak interaction with Ile184 ${ }^{\mathrm{EL} 1.52}$; while the aligned $\operatorname{Trp}^{\mathrm{EL} 1.50}$ of $\mathrm{D}_{3} R$ and $\operatorname{Trp} 101^{\mathrm{EL} 1.50}$ in $D_{4} R$ are stabilized by their interactions with the disulfide bond - their passages towards the position of $\operatorname{Trp} 100^{\mathrm{EL} 1.50}$ in $\mathrm{D}_{2} \mathrm{R}$ are blocked by the extended EL2. In our simulations, Trp100 ${ }^{\mathrm{EL} 1.50}$ in $D_{2} R$ shows significant flexibility and can adopt multiple positions and orientations in $D_{2} R /$ risperidone (c), while Trp96 ${ }^{E L 1.50}$ in $D_{3} R$ is highly stable in $D_{3} R /$ eticlopride (d). (e) The $X^{1}$ and $\mathrm{X}^{2}$ dihedral angles of $\operatorname{Trp} 100^{\mathrm{EL} 1.50}$ in the subset of the $\mathrm{D}_{2} \mathrm{R} /$ risperidone simulations in which EL2 is still in a helical conformation (orange), are more widely distributed than those of $\operatorname{Trp} 96^{\mathrm{EL} 1.50}$ in the $D_{3} R$ /eticlopride simulations in which EL2 remains in extended conformations (cyan). These dihedral angle values in the $D_{2} R$ and $D_{3} R$ structures are indicated with the orange and cyan stars, respectively. (f), For the same two sets of simulations in $\mathbf{e}$, the distance between the center of mass (COM) of the sidechain heavy atoms of Trp100 in $D_{2} R$ and the COM of the Ca atoms of the ligand binding site residues (excluding Trp100, see Methods for the list of the residues) has wider distributions than the corresponding distance between Trp96 ${ }^{\mathrm{EL} 1.50}$ in $\mathrm{D}_{3} R$ and its ligand binding site. These distances in the $D_{2} R$ and $D_{3} R$ structures are indicated with the orange and cyan dotted lines, respectively. 


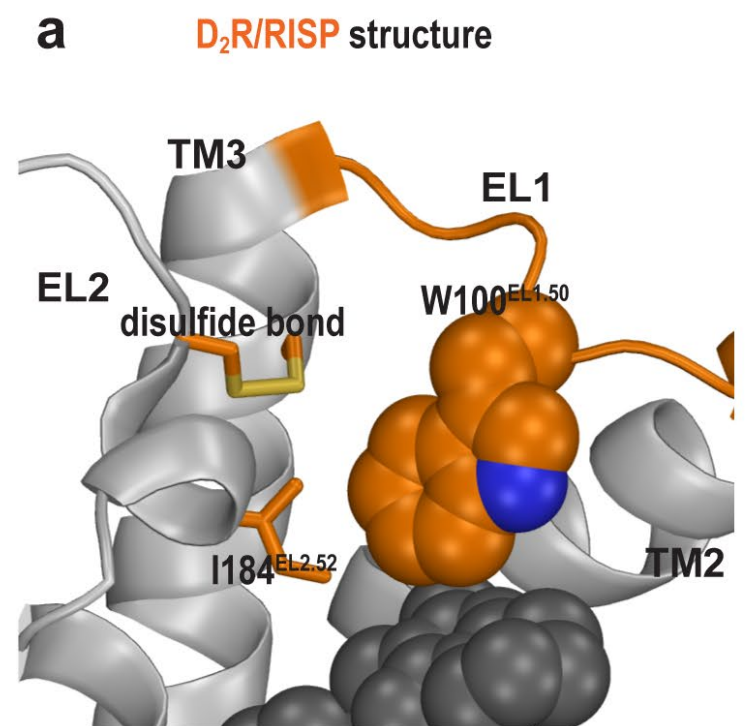

\section{b $\quad D_{3} R / E T Q$ and $D_{4} R / N E M O$ structures}
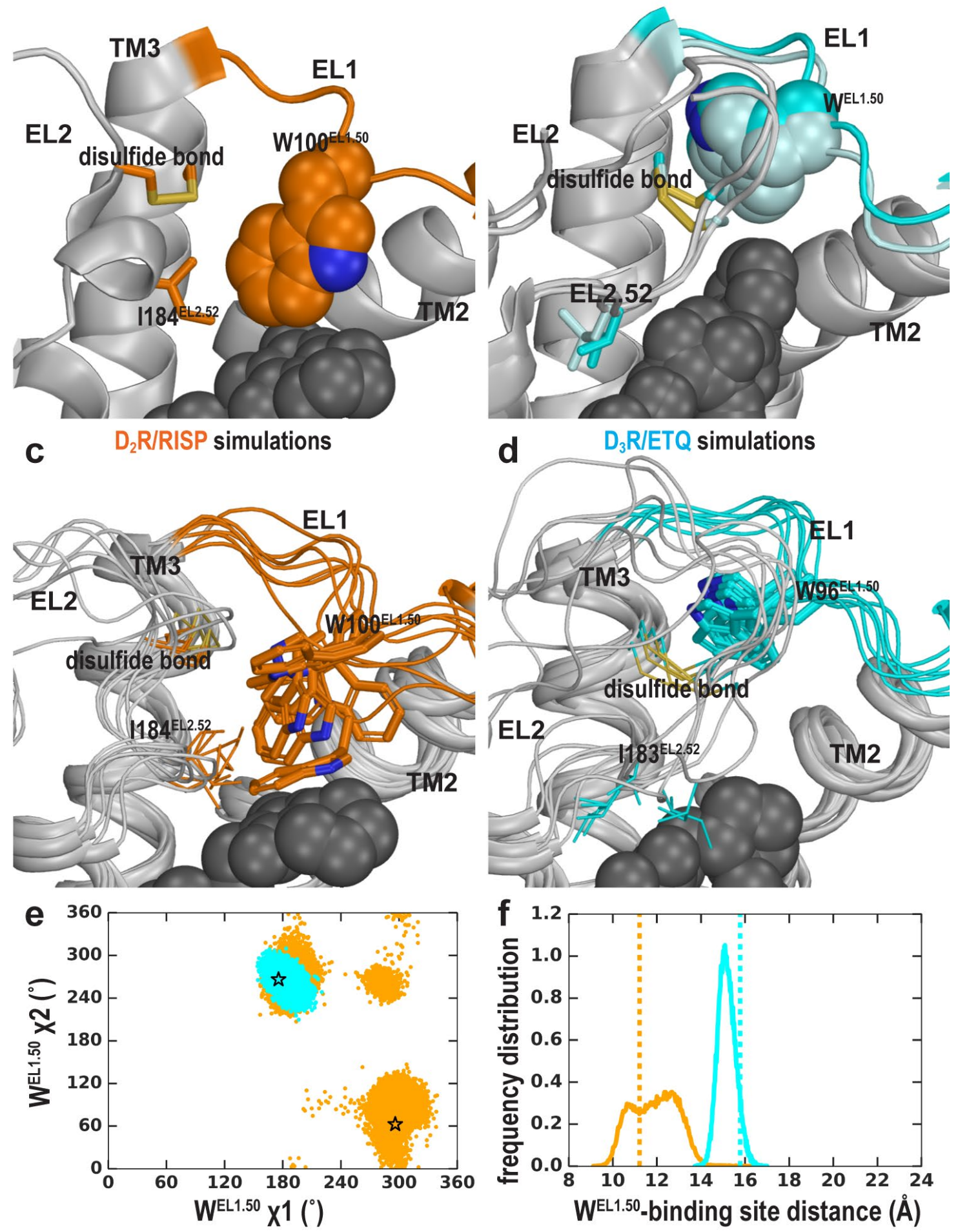
Figure 5. The helical conformation of EL2 in the $D_{2} R /$ risperidone structure has a tendency to unwind in our simulations, regardless of the bound ligand. (a) The Ile183 ${ }^{\mathrm{EL} 2.51}-$ Asn $186^{\mathrm{EL} 2.54}$ backbone $\mathrm{H}$-bond and the Ile183 ${ }^{\mathrm{EL} 2.51}$-Asp $108^{3.26}$ interaction in $\mathrm{D}_{2} \mathrm{R}$ and their aligned interactions in $D_{3} R$. (b) the scatter plots of the two distances in the indicated $D_{2} R$ and $D_{3} R$ complexes. The orange and cyan crosses indicated the distances in the $D_{2} R /$ risperidone and $D_{3} R$ /eticlopride structures, respectively. (c) The distributions of the EL2.51-EL2.54 distances in the indicated simulations. These distances were used to evaluate the tendency to unwind using Markov state model (MSM) analysis in d. (d) The MSM analysis of the transition between the helical and extended conformational states of EL2. The area of each disk representing a state is proportional to the equilibrium probability $(\pi)$ in each simulated condition. The values from the maximum likelihood Bayesian Markov model for $\pi$ and transition rates from 500 Bayesian Markov model samples are shown. Thus, EL2 in all the $D_{2} R$ complexes show significant tendencies to unwind, while that in $D_{3} R$ /eticlopride remains extended. 

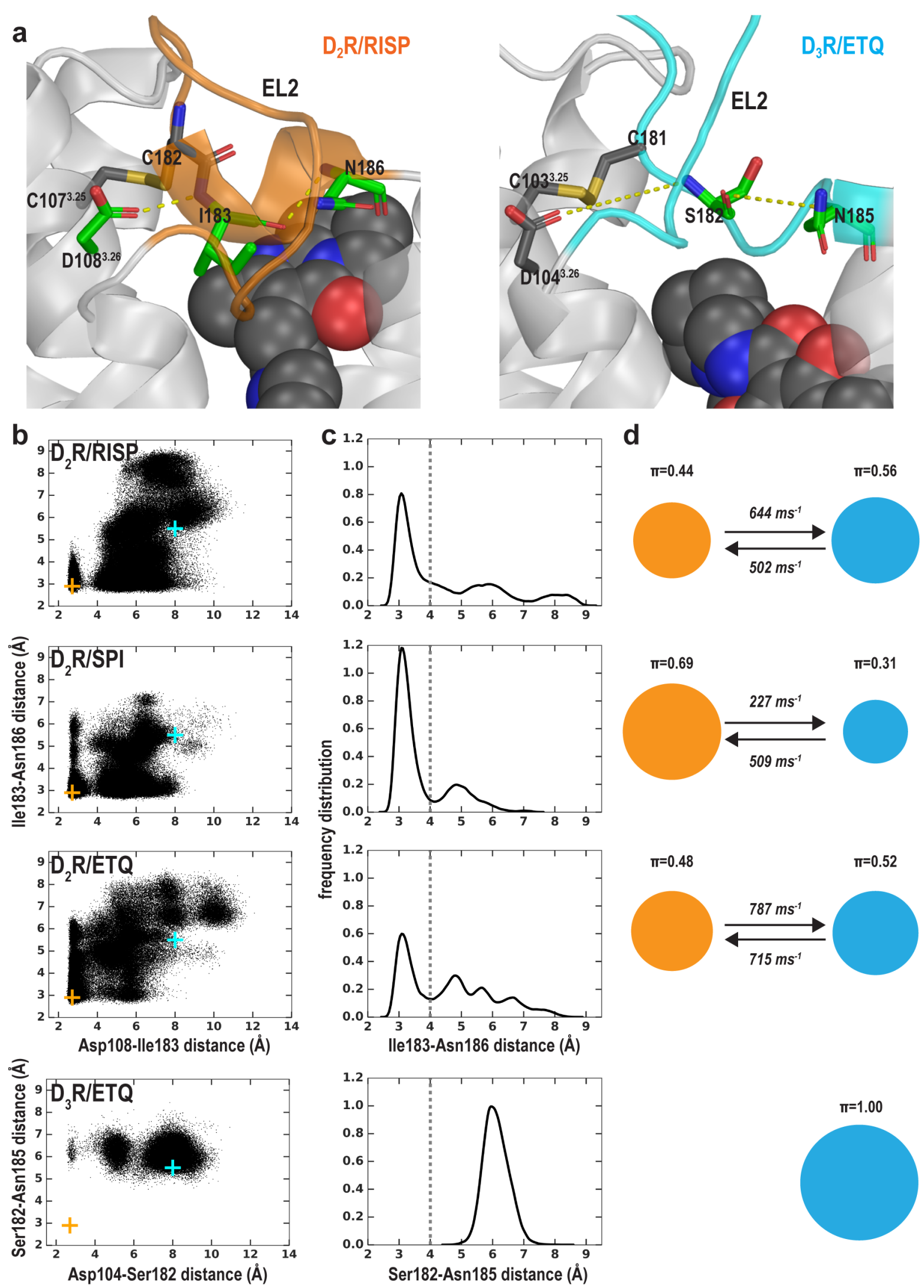


\section{SUPPLEMENTARY INFORMATION}

Supplementary Table 1. Summary of molecular dynamics simulations.

\begin{tabular}{|c|c|c|c|c|c|}
\hline receptor & ligand & $\begin{array}{c}\text { bound } \\
\mathrm{Na}^{+}\end{array}$ & $\begin{array}{c}\text { number of } \\
\text { OPLS3 } \\
\text { trajectories }\end{array}$ & $\begin{array}{l}\text { Number of } \\
\text { CHARMM36 } \\
\text { trajectories }\end{array}$ & $\begin{array}{c}\text { accumulated } \\
\text { simulation time } \\
\text { (ns) }\end{array}$ \\
\hline \multirow{7}{*}{$\mathrm{D}_{2} \mathrm{R}$} & \multirow{2}{*}{ risperidone } & + & 12 & & 29610 \\
\hline & & - & 11 & & 36000 \\
\hline & \multirow{2}{*}{ spiperone } & + & 21 & & 39900 \\
\hline & & - & 17 & & 25470 \\
\hline & \multirow{2}{*}{ eticlopride } & + & 5 & 12 & 46020 \\
\hline & & - & 7 & & 8400 \\
\hline & aripiprazole & + & 40 & & 66660 \\
\hline \multirow{4}{*}{$D_{3} R$} & \multirow{2}{*}{ eticlopride } & + & & 3 & 13200 \\
\hline & & - & & 4 & 6240 \\
\hline & R22 & + & & 7 & 33600 \\
\hline & S22 & - & & 6 & 49920 \\
\hline & & Total & 113 & 32 & 355020 \\
\hline
\end{tabular}


Supplementary Figure 1. Chemical structure alignments of the non-selective $D_{2}$-like receptors ligands.

risperidone

spiperone<smiles>CCc1cc(Cl)c(OC)c(C(=O)NC[C@H]2CCCN2CC)c1O</smiles>

eticlopride

nemonapride 
bioRxiv preprint doi: https://doi.org/10.1101/640870; this version posted May 17, 2019. The copyright holder for this preprint (which was not certified by peer review) is the author/funder. This article is a US Government work. It is not subject to copyright under 17 USC 105 and is also made available for use under a CCO license.

Supplementary Figure 2. Allosteric communication between the $11 e^{3.40}$ sub-pocket and the $\mathrm{Na}^{+}$ binding site. Risperidone (a, b) and spiperone (d, e) similarly occupy the $l l e^{3.40}$ sub-pocket in both the presence and absence of $\mathrm{Na}^{+}$bound at the $\mathrm{Asp} 80^{2.50}$ site. In the eticlopride bound conditions $(\mathbf{g}, \mathbf{h})$, the $l l e^{3.40}$ sub-pocket is not occupied, and Cys ${ }^{3.36}$ shows flexibility in the absence of bound $\mathrm{Na}^{+}$. (c, $\mathbf{f}$, and i) Distributions of the $x^{1}$ rotamer of $C y s^{3.36}$ in the $D_{2} R$ simulations in the presence of different bound ligands.
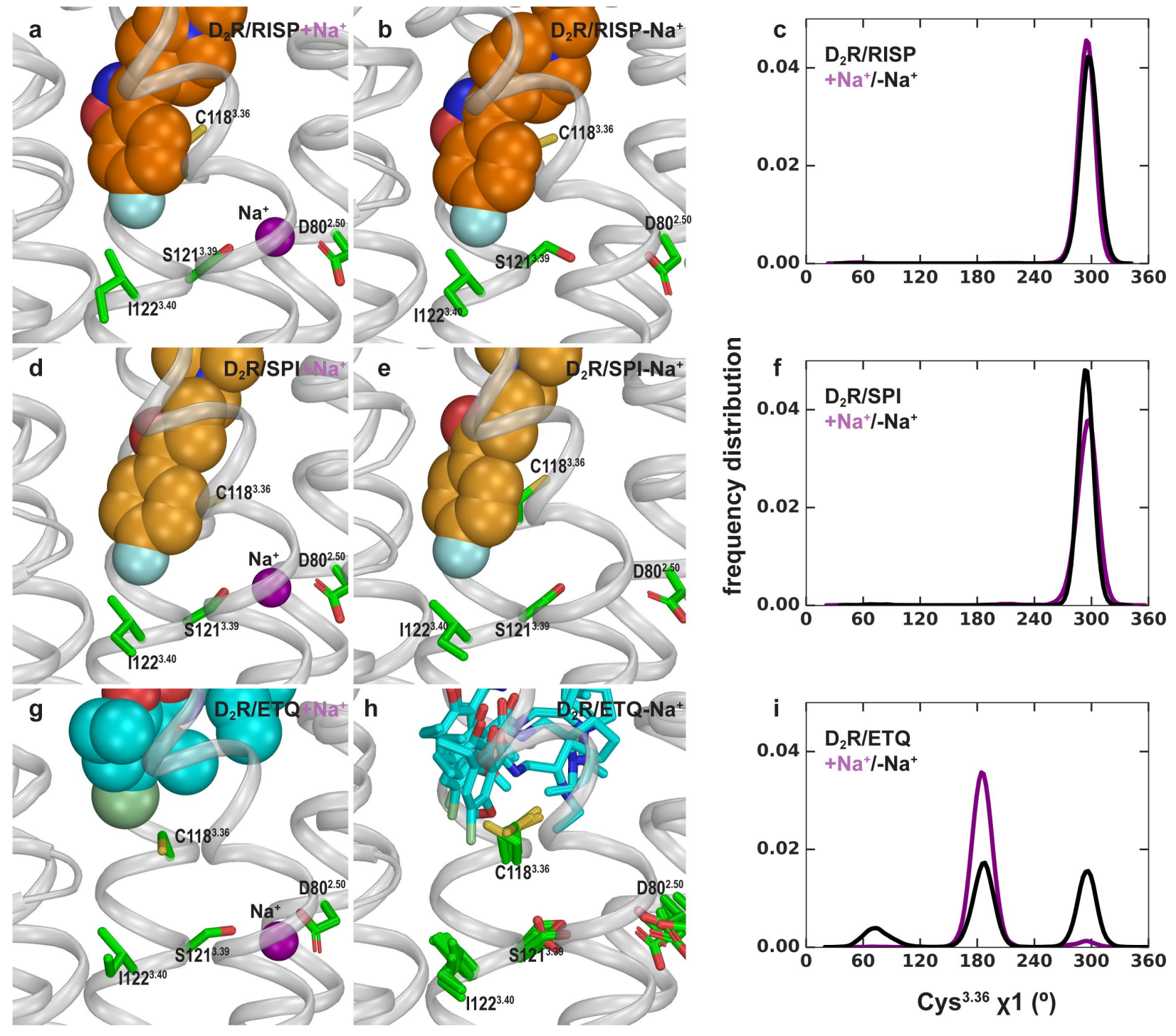
Supplementary Figure 3. Sequence alignment and residue indices of EL1 and EL2 for the receptors being compared in this study. The positions with identical residues are in dark grey shade, the conserved positions are in light grey shade.

$\begin{array}{lrl}\text { EL1 } & & \text { EL1.50 } \\ & & \text { I } \\ \mathrm{D}_{2} \mathrm{R} & 97 & \text { VG-EWKFS } \\ \mathrm{D}_{3} \mathrm{R} & 92 & \text { TGGVWNFS } \\ \mathrm{D}_{4} \mathrm{R} & 97 & \text { QGGAWLLS } \\ 5 \mathrm{HT}_{2 \mathrm{~A}} \mathrm{R} & 137 & \text { YGYRWPLP }\end{array}$

EL2 EL2.40 EL2.50

$D_{2} R \quad 173$ GLNN----ADQNECIIAN

$D_{3} R \quad 171$ GFNTT---GDPTVCSISN

$\mathrm{D}_{4} \mathrm{R} \quad 173$ GLNDV-RGRDPAVCRLED

$5 \mathrm{HT}_{2 \mathrm{~A}} \mathrm{R} 214$ GLQDDSKVFKEGSCLLAD 
Supplementary Figure 4. EL1 is dynamic in the $D_{2} R$ /eticlopride and $D_{2} R /$ spiperone simulations when EL2 is helical. Trp100 shows significant flexibility and can adopt multiple positions and orientations in $D_{2} R$ /eticlopride (a-c) and $D_{2} R /$ spiperone (d-f) simulations. Their $X^{1}$ and $X 2$ dihedral angles of Trp100 (b, e) and the distance between Trp100 and the ligand binding site (c, f) have wide and different distributions. These dihedral angle values in the $D_{2} R$ and $D_{3} R$ structures are indicated with the orange and cyan stars, respectively. The distances in the $D_{2} R$ and $D_{3} R$ structures are indicated with the orange and cyan dotted lines, respectively.
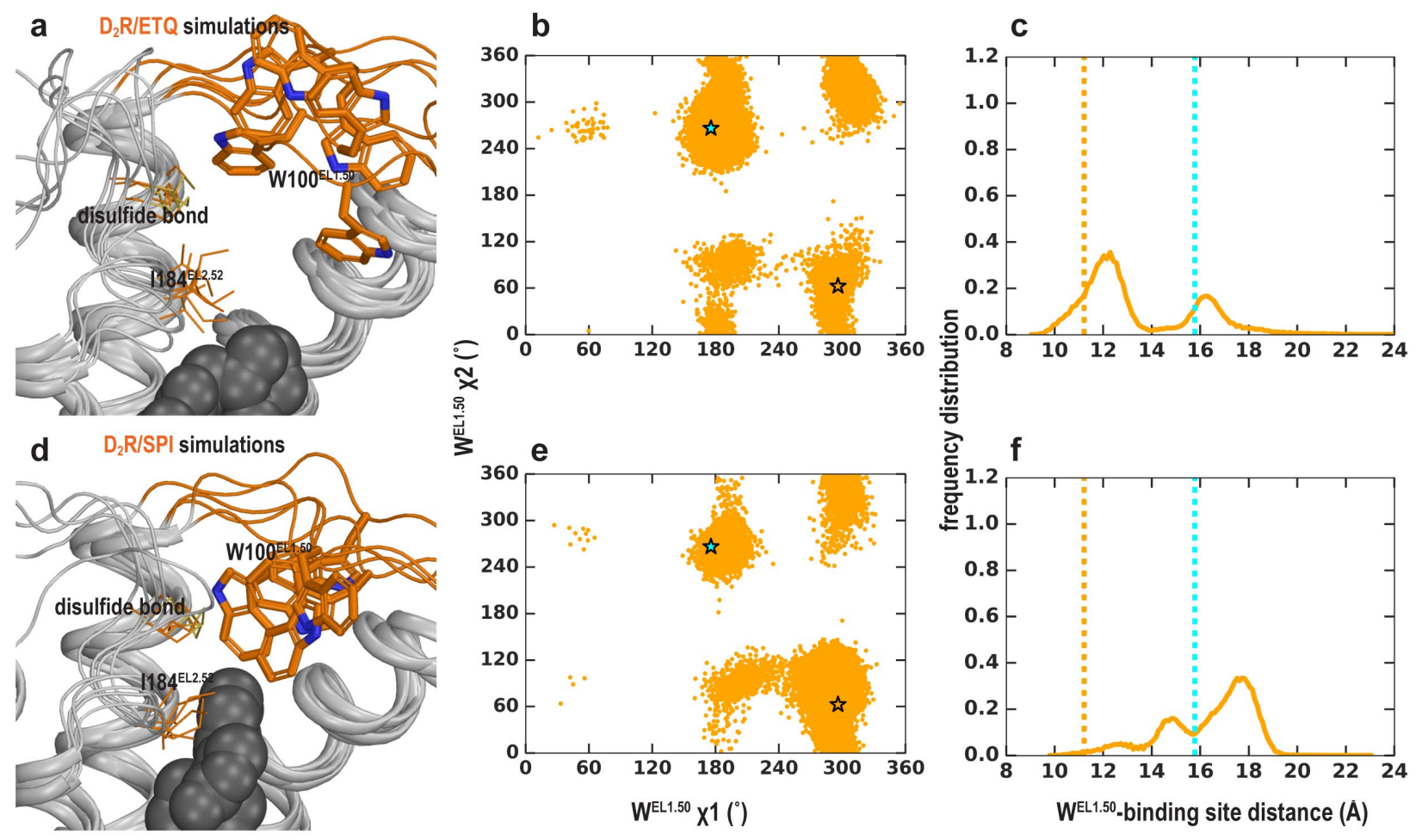
Supplementary Figure 5. The MSM analysis of Ile183-Asn186 distance in the simulations of the $D_{2} R$ /aripiprazole, $D_{3} R / S 22$, and $D_{3} R / R 22$ complexes (Supplementary Table 1 ). The early stage of $D_{3} R / S 22$ and $D_{3} R / R 22$ simulations has been reported previously ${ }^{35}$. The representation and color scheme is the same as that for Fig. 5.

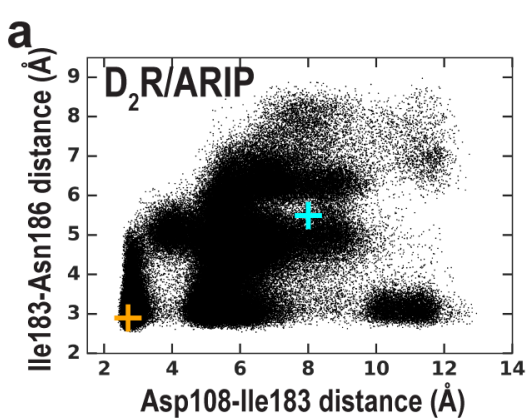

b
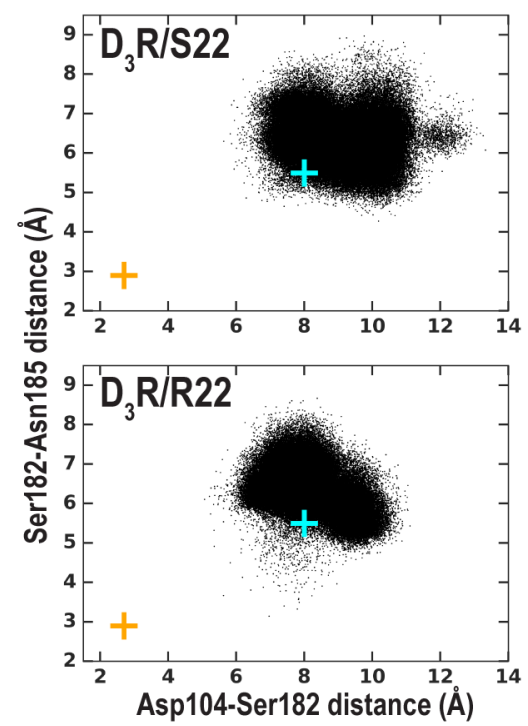

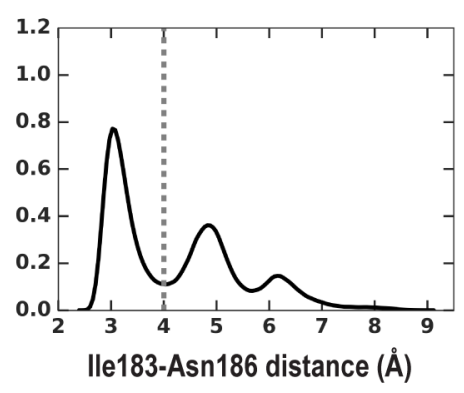

C
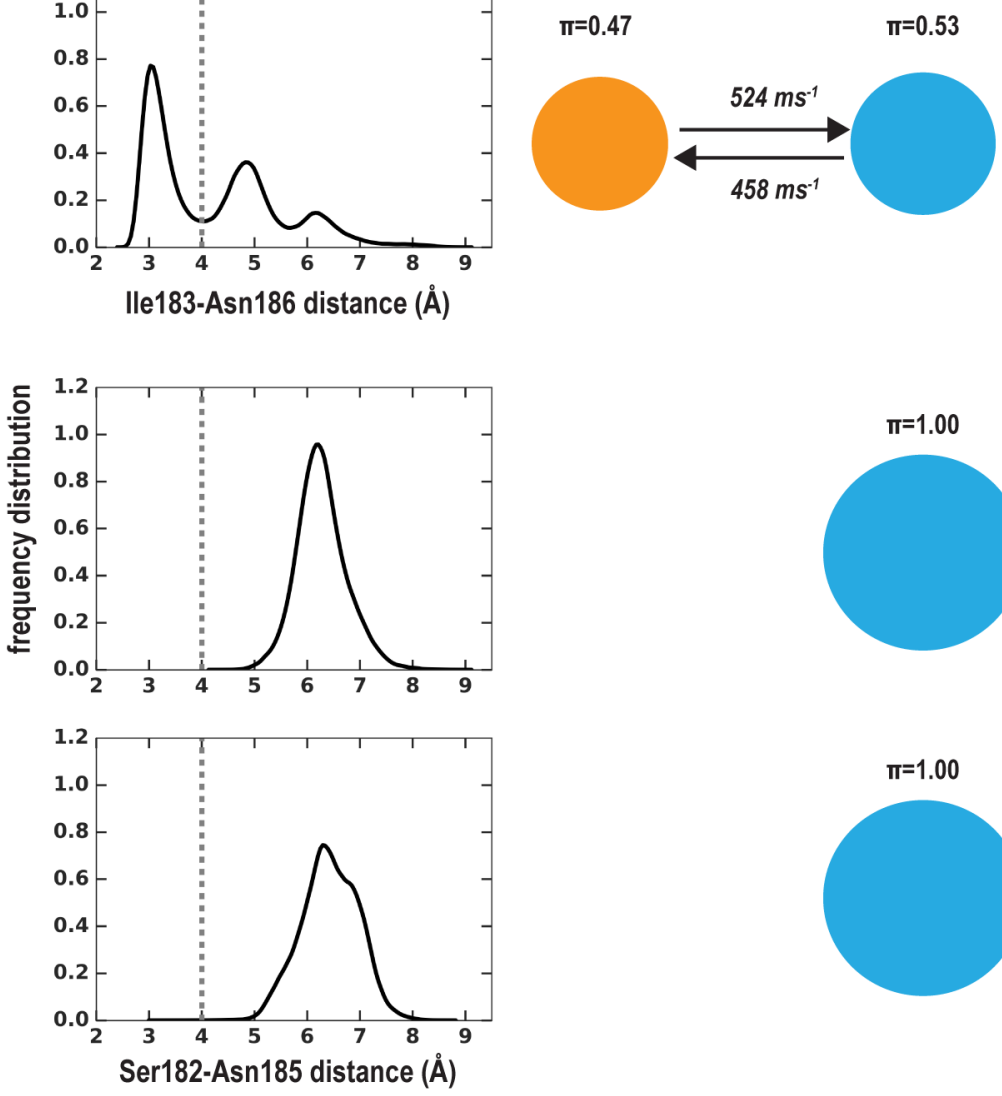

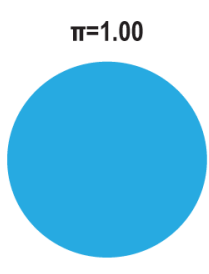

$\pi=1.00$ 
Supplementary Figure 6 . The distinct $D_{2} R$ EL2 conformations revealed by the MD simulations are similar to those of homologous receptors. The C-terminal helical EL2 conformation in the $D_{2} R$ structure (d) can be maintained in the simulations (a). the C-terminal extended conformation (b) is similar to those in the $D_{3} R$ structure $(\mathbf{e})$. The $\mathrm{N}$-terminal helical conformation (c) is reminiscent of that in the $5-\mathrm{HT}_{2 \mathrm{~A}} \mathrm{R} /$ risperidone structure $(\mathbf{g})$, and those in $\beta_{1}$ and $\beta_{2}$ adrenergic receptors structures (not shown).
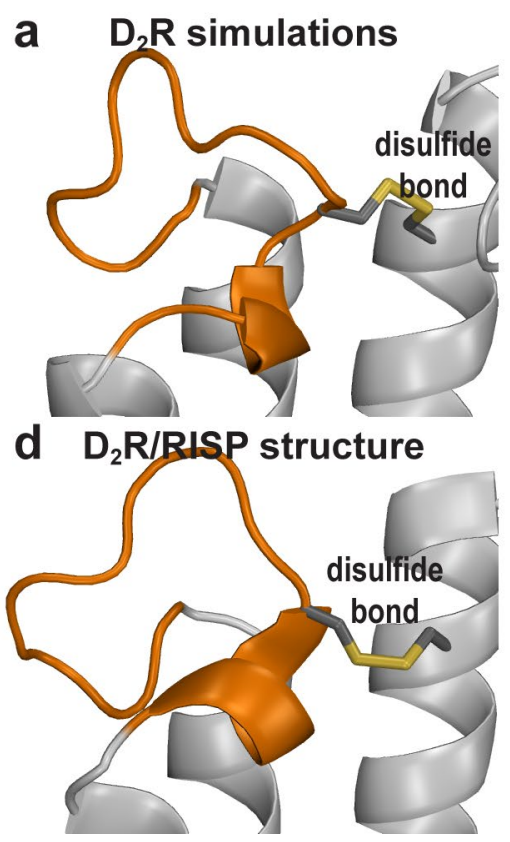

b $\quad D_{2} R$ simulations

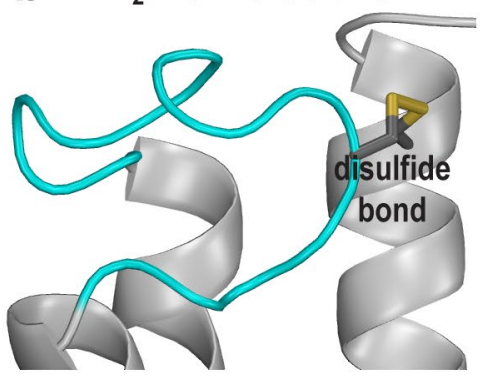

e $D_{3} R / E T Q$ structure

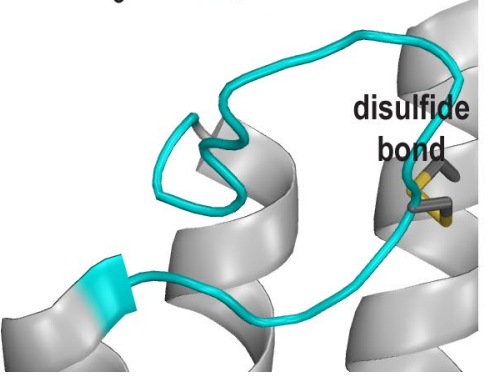

C $\quad D_{2} R$ simulations

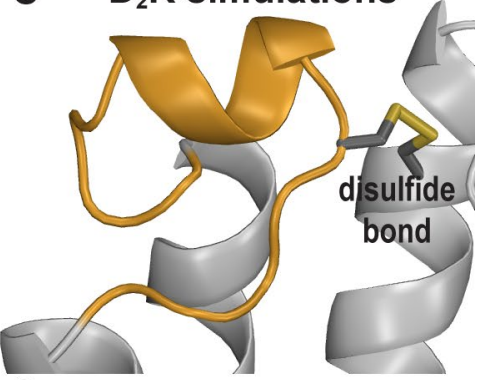

f $5-\mathrm{HT}_{2 \mathrm{~A}} \mathrm{R} / \mathrm{RISP}$ structure

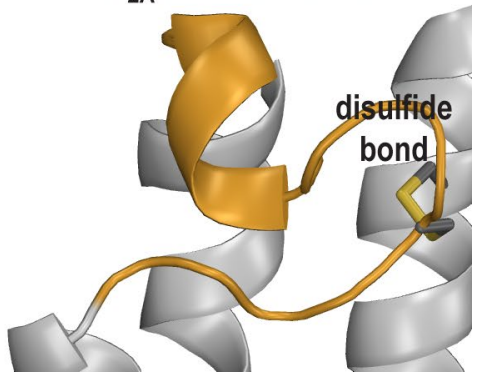


Supplementary Figure 7. The accessibility pattern of EL2 revealed by previous SCAM studies in $D_{2} R$ is more consistent with an extended EL2 conformation similar to that in the $D_{3} R /$ eticlopride structure. The accessible residues are in green, the protected residues are in cyan. In the $D_{2} R /$ risperidone structure (a), lle183 ${ }^{\mathrm{EL} 2.51}$ blocks the accessibility of Gly $173^{\mathrm{EL} 2.37}$ to the OBS, while Asn175 faces lipid. In the $D_{2} R$ /eticlopride simulations (b) and $D_{3} R$ /eticlopride structure (c), Asn ${ }^{\mathrm{EL} 2.39}$ rotates to point inward, while lle $183^{\mathrm{EL} 2.51}$ in $\mathrm{D}_{2} R$ and Ser182 ${ }^{\mathrm{EL} 2.51}$ in $\mathrm{D}_{3} R$ rotates to face the extracellular vestibule of the receptors.
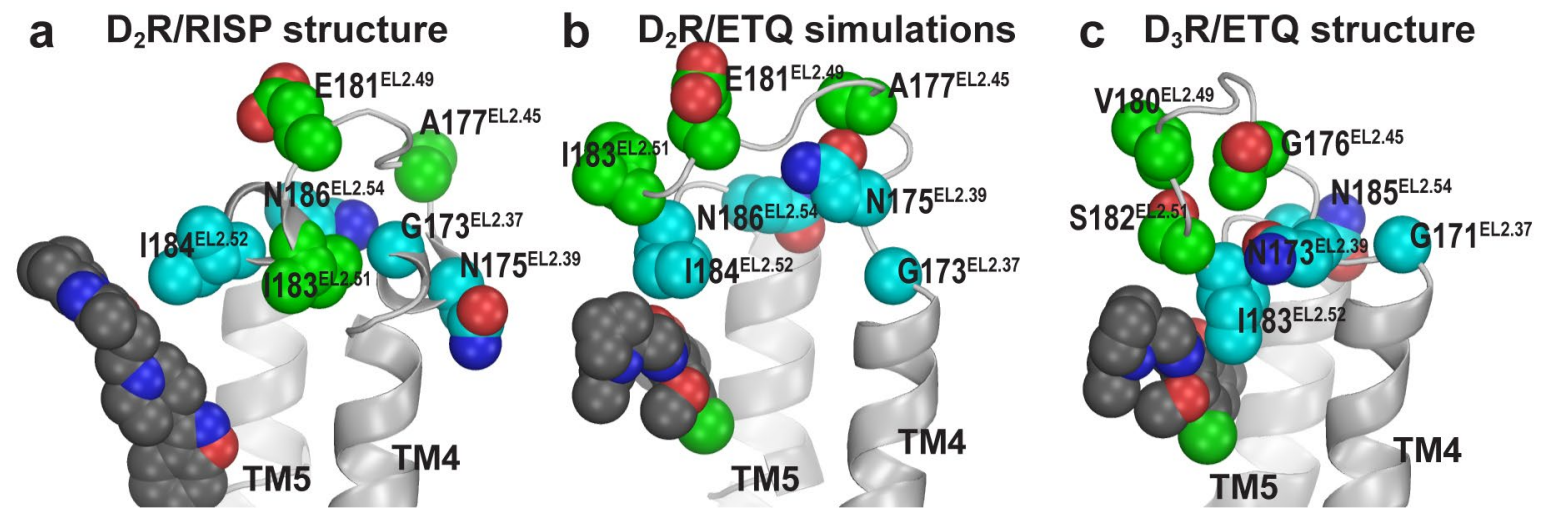
Supplementary Figure 8. Implied timescales (ITS) for the MSM analysis. The implied timescales (ITS) of the transition between the two states in each of the $D_{2} R$ conditions shown in Fig. 5 and Supplementary Fig. 5 are plotted against various lag times. ITSs were not computed for $D_{3} R$ conditions because there was not transition between two states. The ITS of the maximum likelihood Bayesian Markov model is shown in a blue solid line, whereas the means and the $95 \%$ confidence intervals (computed by Bayesian sampling) are shown in dashed and shaded areas, respectively. Timescales smaller than the lag time are shown in grey-shaded area. A lag time of 300 ns was chosen for our analysis.
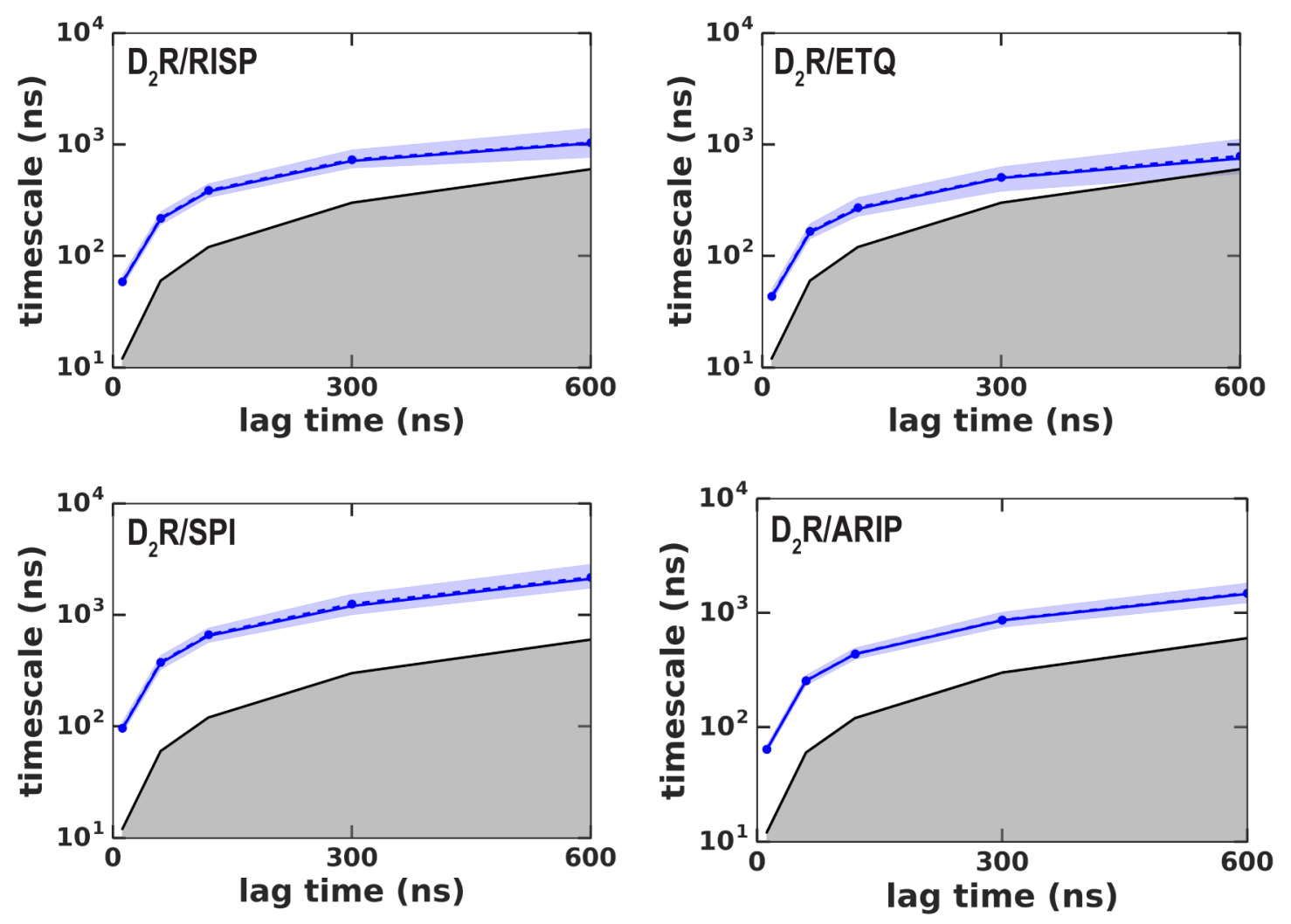
Supplementary Movie 1. A movie of a $4.2 \mu \mathrm{s} \mathrm{D}_{2} \mathrm{R} /$ risperidone trajectory collected using the OPLS3 force field shows spontaneous unwinding of EL2. The conformation of EL2 gradually transitions to an extended configuration similar to that in the D3R structure. See Fig. 3 for the pathway of unwinding. Note that the extended conformation of EL2 stabilizes Trp100 ${ }^{\mathrm{EL} 1.50}$. The Ca atom of Gly $173^{\mathrm{EL} 2.37}$, the sidechains of $\operatorname{Trp} 100^{\mathrm{EL} 1.50}$, Ile $183^{\mathrm{EL} 2.51}$, and Ile $184^{\mathrm{EL} 2.52}$ and the bound risperidone are shown as spheres. Asp $108^{3.26}$ and the disulfide bond between Cys $107^{3.25}$ and Cys $182^{\mathrm{EL} 2.50}$ are shown as sticks. The carbon atoms of Gly $173^{\mathrm{EL} 2.37}$ and Ile184 ${ }^{\mathrm{EL} 2.52}$ are colored in

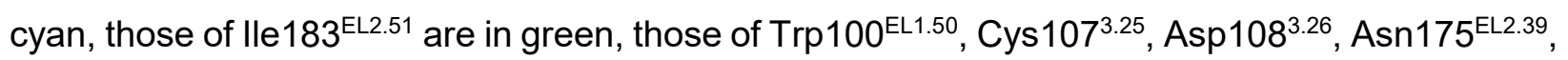
and Cys $182^{\mathrm{EL} 2.50}$ are in dark grey; those of the bound ligand risperidone are in orange.

Supplementary Movie 2. A movie of a $4.2 \mu \mathrm{s} \mathrm{D}_{2}$ R/eticlopride trajectory shows the dynamics of $\operatorname{Trp} 100^{\mathrm{EL} 1.50}$ when the C-terminal portion of EL2 is in a helical conformation. Note that Trp100 ${ }^{\mathrm{EL} 1.50}$ can be stabilized by interacting with the disulfide bond. The presentation and color scheme are similar to those in Supplementary Movie 1, except that the bound carbon atoms of the ligand eticlopride are colored in cyan.

Supplementary Movie 3. A movie of a $3.6 \mu \mathrm{s} \mathrm{D}_{2}$ R/eticlopride trajectory collected using the CHARMM36 force field shows another example of unwinding of EL2. Thus, considering the similar unwinding pathway as that in Movie S1 (Fig. 3), the unwinding does not depend on the force field used in the simulations or the identity of the antagonist bound in the OBS. Note the sidechain of Asn175 ${ }^{\mathrm{EL} 2.39}$ rotates inward and approaches Asp108 3.26 in this trajectory. The presentation and color scheme are the same as those in Supplementary Movie 2.

Supplementary Movie 4. A movie of a $4.5 \mu \mathrm{s} \mathrm{D}_{2} \mathrm{R} /$ risperidone trajectory shows the $\mathrm{N}$-terminal portion of EL2 can transition into a helical conformation when the C-terminal portion is extended. This is a novel EL2 conformation that has not been revealed by the $D_{2} R, D_{3} R$ or $D_{4} R$ structures 
bioRxiv preprint doi: https://doi.org/10.1101/640870; this version posted May 17, 2019. The copyright holder for this preprint (which was not certified by peer review) is the author/funder. This article is a US Government work. It is not subject to copyright under 17 USC 105 and is also made available for use under a CCO license.

but similar to those in the $5-\mathrm{HT}_{2 \mathrm{~A}} \mathrm{R} /$ risperidone, $\beta_{2} \mathrm{AR}$ and $\beta_{2} \mathrm{AR}$ structures (Supplementary Fig.

S6). The presentation and color scheme are the same as those in Supplementary Movie 1. 